\title{
ELLIPTIC OBSTACLE PROBLEMS WITH MEASURE DATA: POTENTIALS AND LOW ORDER REGULARITY
}

\author{
Christoph Scheven
}

\begin{abstract}
We consider obstacle problems with measure data related to elliptic equations of $p$-Laplace type, and investigate the connections between low order regularity properties of the solutions and non-linear potentials of the data. In particular, we give pointwise estimates for the solutions in terms of Wolff potentials and address the questions of boundedness and continuity of the solution.
\end{abstract}

2010 Mathematics Subject Classification: Primary: 35J87, 35B65, 31B35; Secondary: 35R05, 35R06.

Key words: elliptic obstacle problems, $p$-Laplacean, measure data problems, regularity of solutions, non-linear potential theory.

\section{Introduction}

The problems treated in the present work are related to measure data problems of the type

$$
-\operatorname{div} a(x, D u)=\mu \quad \text { on } \Omega,
$$

where the left-hand side is given by an elliptic differential operator of $p$-Laplace type for $p>\frac{2 n}{n+1}$ and $\mu$ is a bounded Radon measure. Such problems have been studied extensively in the literature, see [5], [7], [2], [10] for the foundations of this theory. Interesting examples for solutions to measure data problems are given by super-solutions studied in nonlinear potential theory, see $[\mathbf{1 6}],[\mathbf{2 8}]$. In many applications, e.g. in mechanics or in control theory, elliptic equations are coupled with an obstacle constraint of the form $u \geq \psi$ for a given function $\psi: \Omega \rightarrow \mathbb{R}$, see e. g. [3], [21] for examples and an overview of the classical theory. Another motivation for considering obstacle problems are their applications in non-linear potential theory, where solutions to obstacle problems can be employed very effectively for approximating super-solutions, see e. g. $[\mathbf{1 7}],[\mathbf{2 2}],[\mathbf{1 6}]$. Obstacle problems with measure data have been considered e. g. by Boccardo and Gallouët [6] and by Boccardo and Cirmi [4]. 
In this work, we are interested in the connections between non-linear potentials of the data $\mu$ and $\psi$ and regularity properties of the solutions. The important role of such potentials in the theory of super-solutions has been illuminated in the fundamental work of Kilpeläinen and Malý [19], cf. also the book by Malý and Ziemer [28]. They observed that nonnegative solutions to (1.1) can be estimated in terms of the non-linear Wolff potentials of the right-hand side measure, which are defined as

$$
\mathbf{W}_{\beta, p}^{\mu}\left(x_{0}, R\right):=\int_{0}^{R}\left[\frac{|\mu|\left(B_{\varrho}\left(x_{0}\right)\right)}{\varrho^{n-\beta p}}\right]^{\frac{1}{p-1}} \frac{d \varrho}{\varrho},
$$

whenever $\beta \in(0, n]$. More precisely, Kilpeläinen and Malý derived the following pointwise estimate from above. For every non-negative solution to (1.1) and every ball $B_{R}\left(x_{0}\right) \subset \Omega$, they proved

$$
u\left(x_{0}\right) \leq c\left(\int_{B_{R / 2}\left(x_{0}\right)} u^{\gamma} d x\right)^{\frac{1}{\gamma}}+c \mathbf{W}_{1, p}^{\mu}\left(x_{0}, R\right),
$$

for some $\gamma>p-1$. Moreover, they achieved an estimate from below by the Wolff potential of $\mu$ and a criterion for the continuity of non-negative solutions in terms of the Wolff potential [19], see also [18], [20] for related results. A different method of proof for such estimates has later been developed by Trudinger and Wang [36], who provided extensions to much more general settings. While all of the above-mentioned results are concerned with properties of the solution itself, analogous results for the gradient of solutions have been achieved by Mingione [30] for $p=2$ and by Duzaar and Mingione [11], [13], [14] for general growth exponents. Moreover, their techniques allowed not only to bound the gradient in terms of potentials, but to go one step further and analyze the continuity of the gradient with the help of non-linear potentials [12]. Their approach yields results analogous to the continuity result by Kilpeläinen and Malý mentioned above, but now on the level of the gradient. Finally, we remark that Kuusi and Mingione [23], [24] recently gave an improved criterion for gradient continuity in terms of Riesz potentials, which implies in particular the sharp criterion $\mu \in L(n, 1)$ for gradient continuity also for growth exponents $p>2$.

Gradient estimates for solutions to obstacle problems in terms of potentials have been investigated in our preceding works [32], [35], where we considered more regular vector fields $a(x, \xi)$ that depend Dinicontinuously on $x \in \Omega$ and differentiably on the gradient variable $\xi \in \mathbb{R}^{n}$. In the present work, we are concerned with the question what can be said for much less regular vector fields that need only be Carathéodory 
functions, i. e. measurable in $x$ and continuous in $\xi$. Moreover, contrary to the situation in [35], we are now able to allow an additional divergence term on the right-hand side of (1.1). In such a general situation, regularity of the gradient can not be expected anymore, but one might still ask for local boundedness or continuity of the solution itself. Also for these questions, the techniques developed by Duzaar and Mingione in $[\mathbf{1 1}],[\mathbf{1 2}],[\mathbf{1 4}]$ provide answers in the case of the elliptic equation (1.1). The question of local boundedness can be answered positively with the help of the bound (1.3) by Kilpeläinen and Malý if the Wolff potential $\mathbf{W}_{1, p}^{\mu}(\cdot, R)$ is locally bounded. On the other hand, continuity of the solution to (1.1) follows if the Wolff potential $\mathbf{W}_{1, p}^{\mu}\left(x_{0}, R\right)$ vanishes in the limit $R \searrow 0$, uniformly in $x_{0} \in \Omega^{\prime}$ for all compactly contained subdomains $\Omega^{\prime} \Subset \Omega$ (cf. [19]). The aim of the present work is to investigate the analogous questions for a rather general class of elliptic obstacle problems. Parabolic analogues of these results have been discussed in [32], [33], [34].

\subsection{The setting.}

We investigate solutions to elliptic obstacle problems on a bounded domain $\Omega \subset \mathbb{R}^{n}$ of dimension $n \geq 2$, and more generally suitable limits of solutions as stated in Definition 1.1. Our general assumptions are the following. We suppose that $a: \Omega \times \mathbb{R}^{n} \rightarrow \mathbb{R}^{n}$ is a Carathéodory function, i. e. that $\xi \mapsto a(x, \xi)$ is continuous for a. e. $x \in \Omega$ and that $x \mapsto a(x, \xi)$ is measurable for every $\xi \in \mathbb{R}^{n}$. Moreover, we assume the following standard ellipticity and growth properties for some growth exponent $p \in\left(\frac{2 n}{n+1}, n\right]$ and given structure constants $0<\nu \leq 1 \leq L$ and $s \in[0,1]$.

$$
\begin{aligned}
\left(a(x, \xi)-a\left(x, \xi_{0}\right)\right) \cdot\left(\xi-\xi_{0}\right) & \geq \nu\left(s+|\xi|+\left|\xi_{0}\right|\right)^{p-2}\left|\xi-\xi_{0}\right|^{2}, \\
|a(x, \xi)| & \leq L(1+|\xi|)^{p-1},
\end{aligned}
$$

for all $x \in \Omega$ and $\xi, \xi_{0} \in \mathbb{R}^{n}$. We consider an obstacle function $\psi \in$ $W^{1, p}(\Omega)$. In the classical setting, we consider inhomogeneities $f \in$ $L^{p^{\prime}}(\Omega)$ and $F \in L^{p}\left(\Omega, \mathbb{R}^{n}\right)$ and investigate solutions $u \in W^{1, p}(\Omega)$ with $u \geq \psi$ a. e. on $\Omega$ of the variational inequality

$$
\int_{\Omega} a(x, D u) \cdot D(v-u) d x \geq \int_{\Omega} f(v-u)+|F|^{p-2} F \cdot D(v-u) d x
$$

for all comparison functions $v \in u+W_{0}^{1, p}(\Omega)$ with $v \geq \psi$ a. e. on $\Omega$. The existence of solutions to this inequality with given Dirichlet boundary data follows from classical results, see e. g. [27], [21]. 
However, we are also interested in solutions to obstacle problems with measure data in the sense that we want to replace the inhomogeneity $f \in L^{p^{\prime}}(\Omega)$ appearing in the above formulation of the obstacle problem by a signed Radon measure $\mu$. For this purpose, we introduce the notation $\mathcal{M}_{b}(\Omega)$ for the space of bounded Radon measures, i. e. the space of signed Radon measures $\mu$ whose total variation $|\mu|$ is bounded on $\Omega$ in the sense $|\mu|(\Omega)<\infty$. When considering elliptic problems with general measure data $\mu \in \mathcal{M}_{b}(\Omega)$, it is evident that even in the obstacle-free problem

$$
-\operatorname{div} a(x, D u)=\mu \text { on } \Omega,
$$

we can not expect the existence of solutions in the space $W^{1, p}(\Omega)$ unless the right-hand side satisfies $\mu \in W^{-1, p^{\prime}}(\Omega)$. Therefore, it is not possible to use the formulation (1.6) of the obstacle problem in the case of general measure data. For such more general settings, different notions of solution and corresponding existence results have been developed in the literature. For example, solutions to elliptic obstacle problems with measure data in $L^{1}(\Omega)+W^{-1, p^{\prime}}(\Omega)$ have been constructed in the articles $[\mathbf{6}],[\mathbf{4}],[\mathbf{2 5}]$, based on the concept of entropy solutions (see [5], [2], [8] for the case of obstacle-free problems). In [31], the notion of renormalized solutions has been adapted to the case of obstacle problems, which allows also to treat measures with non-negative singular part. For a brief summary of these results, we refer to our article [35, Section 1.1]. The solutions from the mentioned works can all be constructed by approximating the solution by solutions to regularized obstacle problems for inhomogeneities $f_{i} \in W^{-1, p^{\prime}}(\Omega) \cap L^{1}(\Omega)$ with $f_{i} \stackrel{*}{\rightarrow} \mu$ weakly* in the space $\mathcal{M}_{b}(\Omega)$, as $i \rightarrow \infty$. According to classical theory [27], [21], the approximating obstacle problems have solutions $u_{i} \in W^{1, p}(\Omega)$ in the sense of (1.6) and can be shown to converge to a limit map in a suitable way, cf. [35, Lemma 3.4]. We follow the approach of approximation with regularized problems also for the derivation of our estimates. More precisely, we first derive certain comparison estimates for solutions of the regularized obstacle problems and then pass to the limit. It turns out that the limit maps fulfill the desired potential estimates regardless of the fact if or in what sense they solve the obstacle problems. This is the reason why we state our results not only for solutions, but more generally for limits of approximating solutions in the sense of the following definition. However, the class of functions introduced in the following lemma includes in particular the solutions for obstacle problems with measure data constructed in $[\mathbf{6}],[\mathbf{4}],[\mathbf{2 5}],[\mathbf{3 1}]$, and clearly, also classical solutions of the variational inequality (1.6). 
Definition 1.1. Suppose that $p>1$ and that an obstacle function $\psi \in W^{1, p}(\Omega)$, measure data $\mu \in \mathcal{M}_{b}(\Omega)$, a function $F \in L^{p}\left(\Omega, \mathbb{R}^{n}\right)$ and boundary data $g \in W^{1, p}(\Omega)$ with $g \geq \psi$ a. e. are given. We say that a map $u \in \mathcal{T}_{g}^{1, p}(\Omega)$ (see Section 2 for the definition) with $u \geq \psi$ a. e. on $\Omega$ is a limit of approximating solutions of the obstacle problem $O P(\psi ; \mu, F)$ if there are functions

$$
f_{i} \in W^{-1, p^{\prime}}(\Omega) \cap L^{1}(\Omega) \quad \text { with } \quad f_{i} \stackrel{*}{\rightarrow} \mu \quad \text { in } \mathcal{M}_{b}(\Omega) \text { as } i \rightarrow \infty,
$$

and solutions $u_{i} \in W^{1, p}(\Omega)$ with $u_{i} \geq \psi$ a. e. of the variational inequalities

$$
\int_{\Omega} a\left(x, D u_{i}\right) \cdot D\left(v-u_{i}\right) d x \geq\left\langle f_{i}, v-u_{i}\right\rangle+\int_{\Omega}|F|^{p-2} F \cdot D\left(v-u_{i}\right) d x
$$

for all comparison functions $v \in u_{i}+W_{0}^{1, p}(\Omega)$ with $v \geq \psi$ a. e., such that the maps $u_{i}$ tend to $u$ as $i \rightarrow \infty$ in the sense

$$
\begin{cases}u_{i} \rightarrow u & \text { almost everywhere on } \Omega, \\ \int_{\Omega}\left|u_{i}-u\right|^{q} d x \rightarrow 0 & \text { for every } 0<q<(p-1) \frac{n}{n-p}, \\ \int_{\Omega}\left|D u_{i}-D u\right|^{r} d x \rightarrow 0 & \text { for every } 0<r<(p-1) \frac{n}{n-1} .\end{cases}
$$

The existence of approximating solutions converging in the sense of the above definition follows with standard techniques for measure data problems, see e.g. $[\mathbf{2}],[\mathbf{5}],[\mathbf{7}],[\mathbf{9}]$. A proof tailored to the particular situation considered here can be found in [35, Lemma 3.4].

Remark 1.2. The convergence (1.8) implies $u_{i} \rightarrow u$ in $L^{1}(\Omega)$ as long as $p>\frac{2 n}{n+1}$. Consequently, every limit of approximating solutions in the meaning of the above definition satisfies $u \in L^{1}(\Omega)$ if $p>\frac{2 n}{n+1}$. The gradients of the limits of approximating solutions may not be locally integrable anymore, but they still satisfy $|D u|^{\frac{n}{n+1}} \in L^{1}(\Omega)$ as a consequence of (1.8). These integrability properties are consistent with classical results in measure data problems, which have first been obtained by Lindqvist [26] in the case of $p$-superharmonic functions, see also [16]. The fact that this level of integrability is optimal is already indicated by the simple example of the fundamental solution $u(x):=c|x|^{-\alpha}$, where $\alpha:=\frac{n-p}{p-1}$, of the $p$-Laplacean. This map solves the measure data problem $-\Delta_{p} u=\delta_{0}$ on $\mathbb{R}^{n}$ and satisfies $u \in L_{\text {loc }}^{1}\left(\mathbb{R}^{n}\right)$ if and only if $p>\frac{2 n}{n+1}$. In order to exclude solutions that are not locally integrable, we therefore impose the general assumption $p>\frac{2 n}{n+1}$ throughout this work. 


\subsection{Summary of results.}

We begin by stating our results in the case $p \in[2, n]$. In this case, we prove the following potential estimate for every limit $u \in L^{1}(\Omega)$ of approximating solutions to $O P(\psi ; \mu, F)$, in the sense of the preceding definition. For every Lebesgue point $x_{0} \in \Omega$ of $u$ and every radius $0<R<\min \left\{1, \operatorname{dist}\left(x_{0}, \partial \Omega\right)\right\}$, we have

$$
\begin{aligned}
\left|u\left(x_{0}\right)\right| \leq & c f_{B_{R}\left(x_{0}\right)} 1+|u| d x+c \mathbf{W}_{1, p}^{\mu}\left(x_{0}, R\right) \\
& +c \int_{0}^{R}\left[\frac{|D \psi|^{p}\left(B_{\varrho}\left(x_{0}\right)\right)}{\varrho^{n-p}}\right]^{\frac{1}{p}}+\left[\frac{|F|^{p}\left(B_{\varrho}\left(x_{0}\right)\right)}{\varrho^{n-p}}\right]^{\frac{1}{p}} \frac{d \varrho}{\varrho},
\end{aligned}
$$

with the Wolff potential $\mathbf{W}_{1, p}^{\mu}$ as defined in (1.2) above. Here, we adopted notations such as $|F|^{p}\left(B_{\varrho}\left(x_{0}\right)\right):=\int_{B_{\varrho}\left(x_{0}\right)}|F|^{p} d x$. For the proof, we refer to Theorem 4.3. By classical estimates for potentials, the right-hand side of (1.9) can be bounded further. As an example of the possible applications, we derive criteria for Lorentz space regularity of solutions. In the case of exponents below the duality exponent, i. e. for $r<\left(p^{*}\right)^{\prime}=\frac{n p}{n p-n+p}$, we deduce that

$$
\mu \in L(r, t) \text { implies }|u|^{p-1} \in L_{\mathrm{loc}}\left(\frac{n r}{n-p r}, t\right)
$$

for every $1 \leq t \leq \infty$, together with the corresponding local estimate, see Corollary 4.5. In the case $\left(p^{*}\right)^{\prime}<r<\frac{n}{p}$, this implication remains valid under additional assumptions on $|F|$ and $|D \psi|$. More precisely, in this case we have that

$$
\begin{aligned}
\mu \in L(r, t) \text { and }|F|^{p-1},|D \psi|^{p-1} & \in L\left(\frac{n r}{n-r}, t\right) \\
& \text { implies }|u|^{p-1} \in L_{\mathrm{loc}}\left(\frac{n r}{n-p r}, t\right) .
\end{aligned}
$$

The borderline case $r=\frac{n}{p}$ is particularly interesting, since here one might hope for local boundedness of the solution. This is satisfied by the bound (1.9) if the Wolff potential and the last integral in (1.9) are locally bounded. If more strongly, these quantities tend to zero in the limit $R \searrow 0$, uniformly in $x_{0} \in \Omega^{\prime}$ for every compactly contained subdomain $\Omega^{\prime} \Subset \Omega$, the solutions are even continuous, see Theorem 4.6. This is in particular the case if the data belong to certain Lorentz spaces, which yields the following Lorentz space criterion for continuity, provided $p<n$ (cf. Corollary 4.7).

(1.10) $\mu \in L\left(\frac{n}{p}, \frac{1}{p-1}\right) \quad$ and $|D \psi|,|F| \in L(n, 1) \quad$ implies $\quad u \in C_{\mathrm{loc}}^{0}(\Omega)$. 
Moreover, we also derive a criterion for Hölder continuity of the solution in terms of a Morrey type condition on the data, see Lemma 4.8. To be precise, if the data satisfy

$$
\begin{aligned}
& \sup _{x_{0} \in \Omega^{\prime}} \sup _{0<\varrho<1}\left[\varrho^{p-n-\gamma(p-1)}|\mu|\left(B_{\varrho}\left(x_{0}\right) \cap \Omega\right)\right. \\
&\left.+\varrho^{p-n-\gamma p} \int_{B_{\varrho}\left(x_{0}\right) \cap \Omega}|F|^{p}+|D \psi|^{p} d x\right]<\infty
\end{aligned}
$$

for some $\gamma \in(0,1)$ and every subdomain $\Omega^{\prime} \Subset \Omega$, then we have $u \in$ $C_{\text {loc }}^{0, \alpha}(\Omega)$ for some $\alpha>0$ that can be determined in dependence on $\gamma, n$, $p, \nu$ and $L$.

The proofs are based on a comparison argument, a strategy which has first been used by Mingione [30] for the derivation of potential estimates. All of the above-mentioned results rely on an excess decay estimate for solutions of obstacle problems with measure data. Such excess decay estimates are available for solutions to homogeneous elliptic equations by the DeGiorgi-Nash-Moser theory (cf. Section 4.1). We extend these decay estimates to obstacle problems by a comparison scheme of several steps. In a first step, we remove the right-hand side measure and consider the resulting obstacle problem. In order to derive suitable comparison estimates, we have to make use of truncation methods, as they are standard in the theory of measure data problems (see e.g. [16], [2]). In a second step, we compare with a suitable elliptic equation, whose solutions obey the obstacle condition due to a comparison principle. Finally, in a third step, we compare with the solution of a homogeneous equation to which the DeGiorgi-Nash-Moser theory applies. This procedure yields an excess decay estimate, cf. Lemma 4.2, which can be iterated to give the desired pointwise estimates by potentials of the data, see Theorem 4.3. On the other hand, the mentioned excess decay estimate can be used to examine the continuity of the solutions, as carried out in Theorem 4.6 and Lemma 4.8.

The case $\frac{2 n}{n+1}<p<2$ is more involved since the problem might degenerate for large values of $|D u|$. This causes particularly intricate problems for exponents $p \in\left(\frac{2 n}{n+1}, 2-\frac{1}{n}\right]$, since in this case, the solutions do not in general satisfy $D u \in L^{1}\left(\Omega, \mathbb{R}^{n}\right)$. However, we need some bounds on the gradients in order to control the degeneration, and this is implemented by bounding integrals of the type

$$
f_{B_{\varrho}\left(x_{0}\right)}|D u|^{\frac{n}{n+1}} d x
$$


These integrals turn out to be finite for solutions of measure data problems provided $p>\frac{2 n}{n+1}$. In fact, the solutions that we consider satisfy $|D u|^{p-1} \in L^{\gamma}(\Omega)$ for every $\gamma \in\left[1, \frac{n}{n-1}\right)$, which implies $|D u|^{\frac{n}{n+1}} \in L^{1}(\Omega)$ if and only if $p>\frac{2 n}{n+1}$, cf. Remark 1.2. In order to bound the integrals (1.11) for all of the maps occuring in the comparison argument, we have to derive comparison estimates for the gradient below the $L^{1}$-level, namely on the integrability level $\frac{n}{n+1}$ (cf. Lemma 3.2). For this aim we use once more the truncation arguments from the theory of measure data problems. During the iteration process, we have to deal with additional terms of the type (1.11), but it turns out that most of them can be re-absorbed during the iteration, so that again, we arrive at a potential estimate. However, since the degeneration of the problem depends on the size of the gradient, it seems natural that in this case, the right-hand side contains an integral involving $|D u|$. To be precise, in the case $\frac{2 n}{n+1}<p<2$ the potential estimate reads as follows.

$$
\begin{aligned}
\left|u\left(x_{0}\right)\right| \leq & c f_{B_{R}\left(x_{0}\right)} 1+|u| d x+c R\left(f_{B_{R}\left(x_{0}\right)}|D u|^{\frac{n}{n+1}} d x\right)^{1+\frac{1}{n}} \\
& +c \mathbf{W}_{1, p}^{\mu}\left(x_{0}, R\right)+c \int_{0}^{R}\left[\frac{|D \psi|^{p}\left(B_{\varrho}\left(x_{0}\right)\right)}{\varrho^{n-p}}\right]^{\frac{1}{p}}+\left[\frac{|F|^{p}\left(B_{\varrho}\left(x_{0}\right)\right)}{\varrho^{n-p}}\right]^{\frac{1}{p}} \frac{d \varrho}{\varrho}
\end{aligned}
$$

for all Lebesgue points $x_{0} \in \Omega$ of $u$ and every radius $0<R<\min$ $\left\{1, \operatorname{dist}\left(x_{0}, \partial \Omega\right)\right\}$, see Theorem 4.3. Moreover, the condition (1.10) for the continuity of solutions continues to hold in the same way if $\frac{2 n}{n+1}<p<2$, and also the Lorentz space estimates and the Hölder continuity result are valid analogously for exponents $p<2$, cf. Corollary 4.7 and Lemma 4.8.

\section{Preliminaries}

Notation. In what follows, we consider elliptic obstacle problems on a bounded domain $\Omega \subset \mathbb{R}^{n}$ of dimension $n \geq 2$. For the open ball with radius $\varrho>0$ centered in $x_{0} \in \Omega$ we write $B_{\varrho}\left(x_{0}\right)$, and occasionally we use the shorter notation $B_{\varrho}:=B_{\varrho}(0)$ in case $x_{0}=0$. Given a subset $A \subset \Omega$ and $g \in L^{1}(A)$, we abbreviate $(g)_{A}:=f_{A} g d x$ for the mean value of $g$ over the set $A$. In case $A=B_{\varrho}\left(x_{0}\right)$ is a ball, we write furthermore $(g)_{x_{0}, \varrho}:=(g)_{B_{\varrho}\left(x_{0}\right)}$, respectively $(g)_{\varrho}:=(g)_{B_{\varrho}}$.

Function spaces. We employ the customary notation $W^{1, p}(\Omega)$ for the Sobolev space of weakly differentiable functions $u \in L^{p}(\Omega)$ with $|D u| \in$ $L^{p}(\Omega)$ and write $W_{0}^{1, p}(\Omega)$ for the closure of $C_{\mathrm{cpt}}^{\infty}(\Omega)$ in $W^{1, p}(\Omega)$ with 
respect to the $W^{1, p}$-norm. The dual space to $W_{0}^{1, p}(\Omega)$ will be denoted by $W^{-1, p^{\prime}}(\Omega)$, where $p^{\prime}:=\frac{p}{p-1}$, and for the dual pairing between $W^{-1, p^{\prime}}(\Omega)$ and $W_{0}^{1, p}(\Omega)$ we write $\langle\cdot, \cdot\rangle$. For parameters $1 \leq p<\infty$ and $0<$ $q \leq \infty$, the Lorentz space $L(p, q)(\Omega)$ is defined via the non-increasing rearrangement of a measurable function $f: \Omega \rightarrow \mathbb{R}$ that is defined by

$$
f^{*}:[0, \infty) \rightarrow[0, \infty), \quad f^{*}(s):=\sup \{t \geq 0:|\{|f|>t\}|>s\} .
$$

The space $L(p, q)$ with $0<q<\infty$ consists of those functions $f: \Omega \rightarrow \mathbb{R}$ for which the norms

$$
\|f\|_{L(p, q)}:=\left(\int_{0}^{\infty}\left[r^{1 / p} f^{*}(r)\right]^{q} \frac{d r}{r}\right)^{\frac{1}{q}}
$$

are finite, and the spaces $L(p, \infty)$, also known as Marcinkiewicz spaces, are defined analogously by the condition

$$
\|f\|_{L(p, \infty)}:=\sup _{r>0} r^{1 / p} f^{*}(r)<\infty .
$$

Moreover, we write $L_{\text {loc }}(p, q)(\Omega)$ for the space of functions $f: \Omega \rightarrow \mathbb{R}$ with $\left.f\right|_{\Omega^{\prime}} \in L(p, q)\left(\Omega^{\prime}\right)$ for every compactly contained subdomain $\Omega^{\prime} \subset$ $\Omega$. For a more detailed exposition of the properties of Lorentz spaces we refer to [37].

Natural function spaces for dealing with measure data problems are defined via the truncations

$$
T_{k}(y):= \begin{cases}y & \text { if }|y| \leq k, \\ k \operatorname{sgn}(y) & \text { if }|y|>k,\end{cases}
$$

for $k>0$ and all $y \in \mathbb{R}$. Following [2], we abbreviate

(2.4) $\mathcal{T}^{1, p}(\Omega):=\left\{u: \Omega \rightarrow \mathbb{R}\right.$ measurable: $T_{k}(u) \in W^{1, p}(\Omega)$ for all $\left.k>0\right\}$.

For a discussion of the properties of these spaces we refer to [2]. In particular, functions $u \in \mathcal{T}^{1, p}(\Omega)$ have an almost everywhere well-defined gradient $D u=\Phi$, whose definition is consistent with the weak derivative in case $u \in \mathcal{T}^{1, p}(\Omega) \cap W^{1,1}(\Omega)$. Moreover, for given Dirichlet boundary data $g \in W^{1, p}(\Omega)$, we define

$\mathcal{T}_{g}^{1, p}(\Omega):=\left\{u: \Omega \rightarrow \mathbb{R}\right.$ measurable: $T_{k}(u-g) \in W_{0}^{1, p}(\Omega)$ for all $\left.k>0\right\}$. 
Potentials and Lorentz spaces. We consider parameters $d \geq 1, \gamma \in$ $(0, n]$ and $q>0$. Then we define a general potential of $f \in L^{d}(\Omega)$ by

$$
\mathbf{P}_{\gamma, d, q}^{f}\left(x_{0}, R\right):=\int_{0}^{R}\left[\frac{|f|^{d}\left(B_{\varrho}\left(x_{0}\right)\right)}{\varrho^{n-\gamma}}\right]^{q} \frac{d \varrho}{\varrho},
$$

for $x_{0} \in \mathbb{R}^{n}$ and $R>0$, and $|f|^{d}\left(B_{\varrho}\left(x_{0}\right)\right):=\int_{B_{\varrho}\left(x_{0}\right)}|f|^{d} d x$, where we extended $f$ by zero outside of $\Omega$ for the definition. The above potentials include the localized Riesz potentials

$$
\mathbf{I}_{\gamma}^{f}\left(x_{0}, R\right):=\int_{0}^{R} \frac{|f|\left(B_{\varrho}\left(x_{0}\right)\right)}{\varrho^{n-\gamma}} \frac{d \varrho}{\varrho}
$$

as the special case $d=1=q$, and the non-linear Wolff-potentials defined in (1.2) as the case $d=1$ and $q=\frac{1}{p-1}$. More generally, the potentials $\mathbf{P}_{\gamma, d, q}^{f}$ can be estimated by iterated Riesz potentials of Maz'ja-Havin type as they have been used in the seminal works [1], [29]. Therefore, the above potentials can be estimated by means of classical Riesz potential estimates. Here, we will use the following estimates in Lorentz spaces, a detailed proof of which can be found in [35, Section 5]. Suppose that $d \geq 1,1<r<\infty$ and $1 \leq s \leq \infty$ are given and $f \in L(r d, s d)$. Then, for every $0<\gamma<\frac{n}{r}$ and $0<q \leq 1$ we have

$$
\left\|\mathbf{P}_{\gamma, d, q}^{f}(\cdot, R)\right\|_{L\left(\frac{1}{q} \frac{n r}{n-\gamma r}, \frac{s}{q}\right)} \leq c(n, q, \gamma)\|f\|_{L(r d, s d)}^{q d}
$$

for every $R>0$. In the borderline case $f \in L^{d}(\Omega)$ and $0<\gamma<n$, the following estimate holds true.

$$
\left\|\mathbf{P}_{\gamma, d, q}^{f}(\cdot, R)\right\|_{L\left(\frac{1}{q} \frac{n}{n-\gamma}, \infty\right)} \leq c\|f\|_{L^{d}}^{q d} .
$$

For the proof we refer to [35]. Moreover, we have the following $L^{\infty}$-bound of the potentials $\mathbf{P}_{\gamma, d, q}^{f}$ for every $d \geq 1,0<\gamma<n$ and $q>0$ :

$$
\sup _{x \in \Omega} \mathbf{P}_{\gamma, d, q}^{f}(x, R) \leq c(n, q, \gamma) \int_{0}^{\left|B_{R}\right|}\left[\varrho^{\frac{\gamma}{n d}} f^{*}(\varrho)\right]^{q d} \frac{d \varrho}{\varrho}
$$

for every $R>0$. In particular, we have the implication

$$
f \in L\left(\frac{n d}{\gamma}, q d\right)(\Omega) \quad \Longrightarrow \quad \lim _{R \searrow 0} \sup _{x \in \Omega} \mathbf{P}_{\gamma, d, q}^{f}(x, R)=0 .
$$

The proof has been carried out in $[\mathbf{3 5}]$. 
A comparison principle. The following classical comparison principle will be crucial for constructing comparison maps that satisfy the obstacle constraint $v \geq \psi$ a. e. on $\Omega$. A proof can be retrieved from $[\mathbf{1 6}$, Lemma 3.18].

Lemma 2.1. We assume that $\mathcal{O} \subset \mathbb{R}^{n}$ is a bounded domain and that (1.4) and (1.5) are in force for the vector field $a: \mathcal{O} \times \mathbb{R}^{n} \rightarrow \mathbb{R}^{n}$. Suppose that the maps $v, \psi \in W^{1, p}(\mathcal{O})$ satisfy

$$
\begin{cases}-\operatorname{div} a(\cdot, D v) \geq-\operatorname{div} a(\cdot, D \psi) & \text { on } \mathcal{O} \\ v \geq \psi & \text { on } \partial \mathcal{O}\end{cases}
$$

where the second inequality is to be understood in the sense $(\psi-v)_{+} \in$ $W_{0}^{1, p}(\mathcal{O})$. Then we have $v \geq \psi$ a. e. on $\mathcal{O}$.

Some auxiliary lemmata. The proof of the following well-known lemma can be found e. g. in [15, Lemma 6.1, p. 191].

Lemma 2.2. For $R>0$, let $f:\left[\frac{R}{2}, R\right] \rightarrow[0, \infty)$ be a bounded function satisfying

$$
f(\sigma) \leq \vartheta f(\varrho)+\frac{A}{(\varrho-\sigma)^{\alpha}}+\frac{B}{(\varrho-\sigma)^{\beta}}+C
$$

for all $\frac{R}{2}<\sigma<\varrho<R$ and fixed non-negative constants $A, B, C$, $\alpha>\beta>0$ and a parameter $\vartheta \in(0,1)$. Then we have the estimate

$$
f\left(\frac{R}{2}\right) \leq c(\alpha, \vartheta)\left(\frac{A}{R^{\alpha}}+\frac{B}{R^{\beta}}+C\right) .
$$

The following lemma will be useful for estimating potentials by sums and vice versa.

Lemma 2.3. Assume that $\Phi:(0, R] \rightarrow[0, \infty)$ is a function with the property

$$
\Phi(\varrho) \leq K \Phi(r) \quad \text { if } \theta r \leq \varrho \leq r \leq R,
$$

for constants $K \geq 1$ and $\theta \in(0,1)$. Then the following chain of estimates holds true.

$$
\frac{|\log \theta|}{K} \sum_{\ell=1}^{\infty} \Phi\left(\theta^{\ell} R\right) \leq \int_{0}^{R} \Phi(\varrho) \frac{d \varrho}{\varrho} \leq K|\log \theta| \sum_{\ell=0}^{\infty} \Phi\left(\theta^{\ell} R\right) .
$$

Proof: For $R_{\ell}:=\theta^{\ell} R, \ell \in \mathbb{N}_{0}$, we decompose $(0, R)$ into the intervals $\left(R_{\ell+1}, R_{\ell}\right)$, on which the assumption (2.10) implies the bound $K^{-1} \Phi\left(R_{\ell+1}\right) \leq \Phi \leq K \Phi\left(R_{\ell}\right)$. Summing over $\ell \in \mathbb{N}_{0}$ yields the claim. 


\section{Comparison estimates for measure data problems}

\subsection{Comparison estimates below the growth exponent.}

The following lemma will enable us to derive comparison estimates below the growth exponent by testing with suitable truncations. Results of the same type have been established in [16, Lemma 7.43] and [2, Lemma 4.1]. The particular form of the lemma that we present here was proven in [35].

Lemma 3.1. For fixed constants $M>0, \alpha \in(0,1]$ and $p \in(1, n]$, we suppose that the map $h \in \mathcal{T}_{0}^{1, p}\left(B_{R}\left(x_{0}\right)\right)$ satisfies

$$
\int_{B_{R}\left(x_{0}\right) \cap\{|h| \leq k\}}|D h|^{p} d x \leq M k^{\alpha} \quad \text { for every } k>0 .
$$

(i) For every $0<q<(p-\alpha) \frac{n}{n-p}$, the bound

$$
f_{B_{R}\left(x_{0}\right)}|h|^{q} d x \leq c(n, p, q, \alpha)\left[\frac{M}{R^{n-p}}\right]^{\frac{q}{p-\alpha}}
$$

holds true. In the case $p=n$, this estimate is valid for every $q<\infty$.

(ii) In the case $0<r<(p-\alpha) \frac{n}{n-\alpha}$, we additionally have

$$
f_{B_{R}\left(x_{0}\right)}|D h|^{r} d x \leq c(n, p, r, \alpha)\left[\frac{M}{R^{n-\alpha}}\right]^{\frac{r}{p-\alpha}} .
$$

Our first goal in this section is a suitable comparison estimate between a solution of a measure data problem and the solution of the corresponding homogeneous obstacle problem. We start with a comparison estimate in the classical setting, i. e. with an inhomogeneity in $W^{-1, p^{\prime}}$.

Lemma 3.2. Assume that $f \in W^{-1, p^{\prime}}\left(B_{R}\right) \cap L^{1}\left(B_{R}\right), F \in L^{p}\left(B_{R}, \mathbb{R}^{n}\right)$, and that $u \in W^{1, p}\left(B_{R}\right)$ with $u \geq \psi$ solves the variational inequality

$$
\int_{B_{R}} a(\cdot, D u) \cdot D(v-u) d x \geq\langle f, v-u\rangle+\int_{B_{R}}|F|^{p-2} F \cdot D(v-u) d x
$$

for all $v \in u+W_{0}^{1, p}\left(B_{R}\right)$ with $v \geq \psi$ a. e., where (1.4) and (1.5) are in force. Moreover, let $w \in u+W_{0}^{1, p}\left(B_{R}\right)$ with $w \geq \psi$ be the weak solution of the obstacle problem

$$
\int_{B_{R}} a(\cdot, D w) \cdot D(v-w) d x \geq \int_{B_{R}}|F|^{p-2} F \cdot D(v-w) d x
$$


for all $v \in u+W_{0}^{1, p}\left(B_{R}\right)$ with $v \geq \psi$. In the case $2 \leq p \leq n$, we have the comparison estimate

$$
f_{B_{R}}|D u-D w| d x \leq c\left(R f_{B_{R}}|f| d x\right)^{\frac{1}{p-1}},
$$

while for $\frac{2 n}{n+1}<p<2$, we have the zero order estimate

$$
\begin{aligned}
f_{B_{R}} \mid u & -w \mid d x+R\left(f_{B_{R}}|D u-D w|^{\frac{n}{n+1}} d x\right)^{1+\frac{1}{n}} \\
\leq & c\left(R^{p} f_{B_{R}}|f| d x\right)^{\frac{1}{p-1}} \\
& +c R^{2-p}\left(f_{B_{R}}(s+|D u|)^{\frac{n}{n+1}} d x\right)^{\left(1+\frac{1}{n}\right)(2-p)} R^{p} f_{B_{R}}|f| d x .
\end{aligned}
$$

In both estimates, the constant $c$ depends at most on $n, p$ and $\nu$.

Proof: We point out that it is not possible to use $u$ respectively $w$ as comparison functions in the variational inequalities since these functions might not be bounded, and consequently, this approach would result in comparison estimates involving stronger norms than the $L^{1}$-norm of the inhomogeneity $f$. Therefore, we use the truncated functions $v_{k}:=$ $u+T_{k}(w-u) \in u+W_{0}^{1, p}\left(B_{R}\right)$, for $k>0$, as comparison functions in the variational inequality (3.1). This is allowed since the maps $v_{k}$ satisfy the obstacle constraint $v_{k} \geq \psi$ as a consequence of $u, w \geq \psi$. We thereby get

$$
\begin{aligned}
-f_{B_{R}} a(x, D u) \cdot D\left[T_{k}(w-u)\right] d x \leq & k f_{B_{R}}|f| d x \\
& -f_{B_{R}}|F|^{p-2} F \cdot D\left[T_{k}(w-u)\right] d x
\end{aligned}
$$

where we used $\left|v_{k}-u\right| \leq k$, by the definition of the truncations $v_{k}$. Similarly, we may use $\widetilde{v}_{k}:=w-T_{k}(w-u) \in u+W_{0}^{1, p}\left(B_{R}\right)$, for $k>0$, as comparison function in the inequality (3.2), which gives

$$
f_{B_{R}} a(x, D w) \cdot D\left[T_{k}(w-u)\right] d x \leq f_{B_{R}}|F|^{p-2} F \cdot D\left[T_{k}(w-u)\right] d x
$$


Adding the two preceding inequalities, we arrive at

$$
\int_{B_{R}}[a(x, D w)-a(x, D u)] \cdot D\left[T_{k}(w-u)\right] d x \leq k \int_{B_{R}}|f| d x .
$$

Abbreviating $D_{k}:=\left\{x \in B_{R}:|w(x)-u(x)| \leq k\right\}$, and applying the monotonicity assumption (1.4) on $a$, we thus deduce

$$
\frac{\nu}{c(p)} \int_{D_{k}}(s+|D w|+|D u|)^{p-2}|D w-D u|^{2} d x \leq k \int_{B_{R}}|f| d x .
$$

In order to bound the left-hand side from below, we have to distinguish between two cases.

Case 1: $2 \leq p \leq n$. In this case, the bound (3.8) readily implies

$$
\frac{\nu}{c(p)} \int_{D_{k}}|D u-D w|^{p} d x \leq k \int_{B_{R}}|f| d x
$$

for all $k>0$. An application of Lemma 3.1 with $h:=w-u, \alpha=1$, and $M:=\int_{B_{R}}|f| d x$ now yields the claim (3.3).

Case 2: $2-\frac{1}{n}<p<2$. Because of $p>\frac{2 n}{n+1} \geq \frac{n+2}{n+1}$, the exponent $r:=\frac{n}{n+1}$ satisfies $r>2-p$ and consequently, the exponent $\gamma:=\frac{2 r}{2-p+r}$ satisfies $\gamma \in(1,2)$. Applying Hölder's inequality with the exponents $\frac{2 r}{\gamma(2-p)}$ and $\frac{2}{\gamma}$, we infer

$$
\begin{aligned}
\int_{D_{k}}|D u-D w|^{\gamma} d x \leq & \left(\int_{D_{k}}(s+|D u|+|D w|)^{r} d x\right)^{\frac{2-p}{r} \frac{\gamma}{2}} \\
& \times\left(\int_{D_{k}}(s+|D u|+|D w|)^{p-2}|D u-D w|^{2} d x\right)^{\frac{\gamma}{2}} \\
\leq & c k^{\frac{\gamma}{2}}\left(\int_{B_{R}}(s+|D u|+|D w|)^{r} d x\right)^{\frac{2-p}{r} \frac{\gamma}{2}}\left(\int_{B_{R}}|f| d x\right)^{\frac{\gamma}{2}},
\end{aligned}
$$

by an application of (3.8) in the last step. Next, we wish to apply Lemma 3.1 with $p$ replaced by $\gamma$, the choices $\alpha=\frac{\gamma}{2}, q=1$ and $r=\frac{n}{n+1}$ as well as

$$
M:=c\left(\int_{B_{R}}(s+|D u|+|D w|)^{r} d x\right)^{\frac{2-p}{r} \frac{\gamma}{2}}\left(\int_{B_{R}}|f| d x\right)^{\frac{\gamma}{2}} .
$$


In order to apply the lemma, one has to check the assumptions $1=q<$ $\left(\gamma-\frac{\gamma}{2}\right) \frac{n}{n-\gamma}$ and $r<\left(\gamma-\frac{\gamma}{2}\right) \frac{n}{n-\gamma / 2}$, but these are equivalent to $\gamma>\frac{2 n}{n+2}$, which in turn is equivalent to our assumption $p>\frac{2 n}{n+1}$. Therefore, Lemma 3.1 yields the estimate

$$
\begin{aligned}
f_{B_{R}} \mid & u-w \mid d x+R\left(f_{B_{R}}|D u-D w|^{r} d x\right)^{\frac{1}{r}} \\
\leq & {\left[\frac{M}{R^{n-\gamma}}\right]^{\frac{2}{\gamma}} } \\
& =c R^{2-p}\left(f_{B_{R}}(s+|D u|+|D w|)^{r} d x\right)^{\frac{2-p}{r}} R^{p} f_{B_{R}}|f| d x .
\end{aligned}
$$

In the penultimate integral, we use the estimate $|D w| \leq|D u|+|D u-D w|$ and apply Young's inequality with exponents $\frac{1}{2-p}$ and $\frac{1}{p-1}$ in order to derive the bound

$$
\begin{aligned}
f_{B_{R}} \mid u & -w \mid d x+R\left(f_{B_{R}}|D u-D w|^{r} d x\right)^{\frac{1}{r}} \\
\leq & \frac{1}{2} R\left(f_{B_{R}}|D u-D w|^{r} d x\right)^{\frac{1}{r}}+c\left(R^{p} f_{B_{R}}|f| d x\right)^{\frac{1}{p-1}} \\
& +c R^{2-p}\left(f_{B_{R}}(s+|D u|)^{r} d x\right)^{\frac{2-p}{r}} R^{p} f_{B_{R}}|f| d x .
\end{aligned}
$$

This yields the last claim (3.4) after re-absorbing the first integral from the right-hand side.

Since the comparison estimates in the preceding theorem depend only on the $L^{1}$-norm of the inhomogeneity $f$, they imply also comparison estimates for measure data problems as follows.

Corollary 3.3. Let $w$ be as in the preceding Lemma 3.2, but now let $\mu \in \mathcal{M}_{b}(\Omega)$ and $u$ be a limit of approximating solutions for $O P(\psi ; \mu, F)$, in the sense of Definition 1.1. Then we have the following comparison estimates on every ball $B_{R} \Subset \Omega$, where we write $c$ for constants that depend only on $n, p$ and $\nu$.

$$
f_{B_{R}}|D u-D w| d x \leq c\left[\frac{|\mu|\left(\overline{B_{R}}\right)}{R^{n-1}}\right]^{\frac{1}{p-1}}
$$


if $p \geq 2$, while in the case $\frac{2 n}{n+1}<p<2$, the corresponding statement reads

$$
\begin{aligned}
f_{B_{R}} \mid u & -w \mid d x+R\left(f_{B_{R}}|D u-D w|^{\frac{n}{n+1}} d x\right)^{1+\frac{1}{n}} \\
\leq & c\left[\frac{|\mu|\left(\overline{B_{R}}\right)}{R^{n-p}}\right]^{\frac{1}{p-1}} \\
& +c R^{2-p}\left(f_{B_{R}}(s+|D u|)^{\frac{n}{n+1}} d x\right)^{\left(1+\frac{1}{n}\right)(2-p)} \frac{|\mu|\left(\overline{B_{R}}\right)}{R^{n-p}}
\end{aligned}
$$

Proof: By Definition 1.1, there are functions $f_{i} \in W^{-1, p^{\prime}}\left(B_{R}\right) \cap L^{1}\left(B_{R}\right)$ with $f_{i} \stackrel{*}{\rightarrow} \mu$ weakly* in $\mathcal{M}_{b}\left(B_{R}\right)$ and solutions $u_{i} \in W^{1, p}\left(B_{R}\right)$ of the obstacle problems (1.7) with $u_{i} \rightarrow u$ in the sense of (1.8), as $i \rightarrow \infty$. We begin by considering the case $p>2-\frac{1}{n}$, which is equivalent to $(p-1) \frac{n}{n-1}>1$. In this case, the convergence (1.8) includes in particular the convergence $u_{i} \rightarrow u$ in $W^{1,1}\left(B_{R}\right)$. Moreover, the weak*-convergence $f_{i} \stackrel{*}{\rightarrow} \mu$ in $\mathcal{M}_{b}\left(B_{R}\right)$ implies

$$
\limsup _{i \rightarrow \infty} \int_{B_{R}}\left|f_{i}\right| d x \leq|\mu|\left(\overline{B_{R}}\right) .
$$

Therefore, the claim (3.9) follows by applying the comparison estimates from Lemma 3.2 to the solutions $u_{i}$ of the regularized problems and passing to the limit $i \rightarrow \infty$. For the proof of the last claim (3.10), we note that for exponents with $\frac{2 n}{n+1}<p<2$, the convergence (1.8) implies in particular $u_{i} \rightarrow u$ in $L^{1}\left(B_{R}\right)$ and

$$
\int_{B_{R}}\left|D u_{i}-D u\right|^{\frac{n}{n+1}} d x \rightarrow 0,
$$

as $i \rightarrow \infty$, since the assumption $p>\frac{2 n}{n+2}$ implies $1<(p-1) \frac{n}{n-p}$ and $\frac{n}{n+1}<(p-1) \frac{n}{n-1}$. Thus, the last asserted estimate follows from the estimate (3.4) for $u_{i}$ by passing to the limit.

\subsection{Comparison estimates at the level of the growth exponent.}

The proof of the potential estimates also contains some estimates involving $L^{p}$-norms of the gradients of solutions. These will be matched with the comparison estimates on $L^{1}$-level from the preceding paragraph by the following reverse Hölder inequality. 
Lemma 3.4. Assume that $w \in W^{1, p}\left(B_{R}\right)$ with $w \geq \psi$ a. e. on $B_{R}$ solves the variational inequality

$$
\int_{B_{R}} a(x, D w) \cdot D(v-w) d x \geq \int_{B_{R}}|F|^{p-2} F \cdot D(v-w) d x
$$

for all comparison functions $v \in w+W_{0}^{1, p}\left(B_{R}\right)$ with $v \geq \psi$ a. e. on $B_{R}$, where the assumptions (1.4) and (1.5) are valid for a growth exponent $p>1$. Then, the solution satisfies the reverse Hölder inequality

$$
f_{B_{R / 2}}|D w|^{p} d x \leq c\left(f_{B_{R}}|D w|^{r} d x\right)^{\frac{p}{r}}+c f_{B_{R}} 1+|D \psi|^{p}+|F|^{p} d x
$$

for every exponent $r \in(0, p)$, with a constant $c=c(n, p, r, \nu, L)$.

Proof: For arbitrary radii $\sigma$ and $\varrho$ with $\frac{R}{2}<\sigma<\varrho<R$, we choose a cut-off function $\zeta \in C_{0}^{\infty}\left(B_{\varrho},[0,1]\right)$ with $\zeta \equiv 1$ on $B_{\sigma}$ and $|D \psi| \leq \frac{2}{\varrho-\sigma}$ on $B_{\varrho}$. We define a comparison function

$v:=w+\zeta^{p}\left[\psi-(\psi)_{\varrho}-\left(w-(w)_{\varrho}\right)\right]=\left(1-\zeta^{p}\right) w+\zeta^{p} \psi+\zeta^{p}\left[(w)_{\varrho}-(\psi)_{\varrho}\right] \geq \psi$.

The obstacle constraint $v \geq \psi$ is satisfied since $w \geq \psi$ and $(w)_{\varrho} \geq$ $(\psi)_{\varrho}$, and moreover we have $v \in w+W_{0}^{1, p}\left(B_{R}\right)$. The function $v$ is thus admissible in the variational inequality for $w$, from which we obtain

$$
\begin{aligned}
0 \leq & \int_{B_{R}}\left[a(x, D w)-|F|^{p-2} F\right] \cdot D\left(\zeta^{p}\left(\psi-(\psi)_{\varrho}-w+(w)_{\varrho}\right)\right) d x \\
= & -\int_{B_{R}} \zeta^{p} a(x, D w) \cdot D w-\zeta^{p}|F|^{p-2} F \cdot D w d x \\
& +\int_{B_{R}}\left[a(x, D w)-|F|^{p-2} F\right] \\
& \quad \times\left[\zeta^{p} D \psi+p \zeta^{p-1} D \zeta \otimes\left(\psi-(\psi)_{\varrho}-w+(w)_{\varrho}\right)\right] d x .
\end{aligned}
$$

By means of the assumptions (1.4) and (1.5) on $a(x, \xi)$ and the properties of $\zeta$, we deduce

$$
\begin{aligned}
f_{B_{R}} \zeta^{p}(s+|D w|)^{p-2}|D w|^{2} d x \\
\leq c f_{B_{\varrho}} \zeta^{p}|D w|+\zeta^{p}|F|^{p-1}|D w| d x \\
\quad+c \int_{B_{R}} \zeta^{p-1}\left[(1+|D w|)^{p-1}+|F|^{p-1}\right] \\
\quad \times\left[|D \psi|+\frac{\left|\psi-(\psi)_{\varrho}\right|}{\varrho-\sigma}+\frac{\left|w-(w)_{\varrho}\right|}{\varrho-\sigma}\right] d x .
\end{aligned}
$$


From this we infer by Young's inequality

$$
\begin{aligned}
f_{B_{R}} \zeta^{p}|D w|^{p} d x \leq & \frac{1}{2} f_{B_{R}} \zeta^{p}|D w|^{p} d x \\
& +c f_{B_{\varrho}} 1+|D \psi|^{p}+|F|^{p}+\left|\frac{\psi-(\psi)_{\varrho}}{\varrho-\sigma}\right|^{p}+\left|\frac{w-(w)_{\varrho}}{\varrho-\sigma}\right|^{p} d x .
\end{aligned}
$$

Here, in the case $p<2$ we distinguished between the cases $|D w| \leq s$ and $|D w|>s$ in order to estimate $|D w|^{p} \leq 1+2^{2-p}(s+|D w|)^{p-2}|D w|^{2}$. Reabsorbing the first term on the right-hand side of the preceding estimate and applying the Poincaré-Sobolev inequality, we arrive at

$$
\begin{aligned}
f_{B_{\sigma}}|D w|^{p} d x \leq & c\left(\frac{\varrho}{\varrho-\sigma}\right)^{p} \\
& \times\left[f_{B_{\varrho}} 1+|D \psi|^{p}+|F|^{p} d x+\left(f_{B_{\varrho}}|D w|^{\frac{n p}{n+p}} d x\right)^{\frac{n+p}{n}}\right] .
\end{aligned}
$$

For the estimate of the last integral, we interpolate the $L^{\frac{n p}{(n+p) r}}$-norm of $|D w|^{r}$ between the $L^{p / r_{-}}$and the $L^{1}$-norm and apply Young's inequality, which leads us to

$$
\begin{aligned}
c\left(\frac{\varrho}{\varrho-\sigma}\right)^{p} & \left(f_{B_{\varrho}}|D w|^{\frac{n p}{n+p}} d x\right)^{\frac{n+p}{n}} \\
& \leq \frac{1}{2} f_{B_{\varrho}}|D w|^{p} d x+c\left(\frac{\varrho}{\varrho-\sigma}\right)^{n \frac{p-r}{r}}\left(f_{B_{\varrho}}|D w|^{r} d x\right)^{\frac{p}{r}} .
\end{aligned}
$$

Joining the last two estimates, we deduce

$$
\begin{aligned}
f_{B_{\sigma}}|D w|^{p} d x \leq & \frac{1}{2} f_{B_{\varrho}}|D w|^{p} d x+c\left(\frac{R}{\varrho-\sigma}\right)^{n \frac{p-r}{r}}\left(f_{B_{R}}|D w|^{r} d x\right)^{\frac{p}{r}} \\
& +c\left(\frac{R}{\varrho-\sigma}\right)^{p} f_{B_{R}} 1+|D \psi|^{p}+|F|^{p} d x .
\end{aligned}
$$

Applying Lemma 2.2, we absorb the first term on the right-hand side, which proves the claim.

Clearly, an analogous statement holds for solutions of an obstacle-free problem: 
Lemma 3.5. Let $w \in W^{1, p}\left(B_{R}\right)$ be a weak solution to the elliptic equation

$$
-\operatorname{div} a(x, D u)=-\operatorname{div}\left(|F|^{p-2} F\right) \quad \text { on } B_{R},
$$

where the assumptions (1.4) and (1.5) are in force. Then, for an arbitrary $r \in(0, p)$ and a constant $c=c(n, p, r, \nu, L)$, the following estimate holds.

$$
f_{B_{R / 2}}|D w|^{p} d x \leq c\left(f_{B_{R}}|D w|^{r} d x\right)^{\frac{p}{r}}+c f_{B_{R}} 1+|F|^{p} d x .
$$

Proof: We test the equation with $\varphi:=\zeta^{p}\left(w-(w)_{\varrho}\right)$, where $\zeta \in C_{0}^{\infty}\left(B_{\varrho}\right)$ is a cut-off function as in the preceding proof. The remainder of the proof is analogous to the preceding one, with the only difference that all terms involving $\psi$ can be omitted.

Lemma 3.6. Under the assumptions (1.4) and (1.5), we assume that $u \in W^{1, p}\left(B_{R}\right)$ with $u \geq \psi$ a. e. is a solution of the variational inequality

$$
\int_{B_{R}} a(x, D u) \cdot D(v-u) d x \geq \int_{B_{R}}|F|^{p-2} F \cdot D(v-u) d x
$$

for all comparison functions $v \in u+W_{0}^{1, p}\left(B_{R}\right)$ with $v \geq \psi$ a. e., and let $w \in u+W_{0}^{1, p}\left(B_{R}\right)$ be the weak solution to the elliptic equation

$$
-\operatorname{div} a(x, D w)=-\operatorname{div} a(x, D \psi) \quad \text { on } B_{R} .
$$

Then, with a constant $c$ depending at most on $p, \nu$ and $L$, the bound

$$
f_{B_{R}}|D u-D w|^{p} d x \leq c f_{B_{R}} 1+|D \psi|^{p}+|F|^{p} d x
$$

holds true in the case $p \geq 2$, while for $1<p<2$, we have the estimate

$$
\begin{aligned}
f_{B_{R}}|D u-D w|^{p} d x \leq & c f_{B_{R}} 1+|D \psi|^{p}+|F|^{p} d x \\
& +c\left(f_{B_{R}} 1+|D u|^{p} d x\right)^{2-p} \\
& \times\left(f_{B_{R}} 1+|D \psi|^{p}+|F|^{p} d x\right)^{p-1} .
\end{aligned}
$$


Proof: We use $v:=w$ as comparison function in the variational inequality (3.11). This is possible because the comparison principle from Lemma 2.1 guarantees $w \geq \psi$ a. e. on $B_{R}$. Moreover, we test the equation (3.12) with $w-u \in W_{0}^{1, p}\left(B_{R}\right)$. This leads us to

$$
\begin{aligned}
f_{B_{R}}[a(x, D w)-a(x, D u)] & \cdot D(w-u) d x \\
\leq & f_{B_{R}}\left[a(x, D \psi)-|F|^{p-2} F\right] \cdot D(w-u) d x .
\end{aligned}
$$

Estimating the left-hand side from below by the monotonicity assumption (1.4) and the right-hand side from above by (1.5) and Hölder's inequality, we deduce

$$
\begin{aligned}
f_{B_{R}} & (s+|D u|+|D w|)^{p-2}|D u-D w|^{2} d x \\
& \leq c\left(f_{B_{R}} 1+|D \psi|^{p}+|F|^{p} d x\right)^{\frac{p-1}{p}}\left(f_{B_{R}}|D u-D w|^{p} d x\right)^{\frac{1}{p}}
\end{aligned}
$$

with a constant $c=c(p, \nu, L)$. In the case $p \geq 2$, we can absorb the last factor into the left-hand side, which readily yields the claim. For exponents $p<2$ however, we estimate

$$
\begin{aligned}
f_{B_{R}}|D u-D w|^{p} d x \leq & c\left(f_{B_{R}}(s+|D u|+|D w|)^{p-2}|D u-D w|^{2} d x\right)^{\frac{p}{2}} \\
& \times\left(f_{B_{R}} 1+|D u|^{p}+|D u-D w|^{p} d x\right)^{\frac{2-p}{2}} \\
\leq & c\left(f_{B_{R}} 1+|D \psi|^{p}+|F|^{p} d x\right)^{\frac{p-1}{2}} \\
& \times\left(f_{B_{R}}|D u-D w|^{p} d x\right)^{\frac{1}{2}}\left(f_{B_{R}} 1+|D u|^{p} d x\right)^{\frac{2-p}{2}} \\
& +c\left(f_{B_{R}} 1+|D \psi|^{p}+|F|^{p} d x\right)^{\frac{p-1}{2}} \\
& \times\left(f_{B_{R}}|D u-D w|^{p} d x\right)^{\frac{3-p}{2}},
\end{aligned}
$$

where we applied the estimate (3.13) in the last step. We bound the right-hand side by Young's inequality, once with the exponent 2, and 
once with exponents $\frac{2}{p-1}$ and $\frac{2}{3-p}$. This gives

$$
\begin{aligned}
f_{B_{R}}|D u-D w|^{p} d x \leq & \frac{1}{2} f_{B_{R}}|D u-D w|^{p} d x+c \int_{B_{R}} 1+|D \psi|^{p}+|F|^{p} d x \\
& +c\left(f_{B_{R}} 1+|D \psi|^{p}+|F|^{p} d x\right)^{p-1}\left(f_{B_{R}} 1+|D u|^{p} d x\right)^{2-p} .
\end{aligned}
$$

Here, we can absorb the first integral on the right-hand side in order to derive the claim in the case $p<2$.

Finally, we have a similar result for the comparison of two equations.

Lemma 3.7. We assume that (1.4) and (1.5) are in force and that $u \in W^{1, p}\left(B_{R}\right)$ is a weak solution of the elliptic equation

$$
-\operatorname{div} a(x, D u)=-\operatorname{div} a(x, D \psi) \quad \text { on } B_{R}
$$

and that $w \in u+W_{0}^{1, p}\left(B_{R}\right)$ weakly solves the corresponding homogeneous equation

$$
-\operatorname{div} a(x, D w)=0 \quad \text { on } B_{R} .
$$

Then, for $p \geq 2$ we have

$$
f_{B_{R}}|D u-D w|^{p} d x \leq c f_{B_{R}} 1+|D \psi|^{p} d x
$$

with a constant $c=c(n, p, \nu, L)$, while for $1<p<2$, we can estimate

$$
\begin{aligned}
f_{B_{R}}|D u-D w|^{p} d x \leq & c f_{B_{R}}|D \psi|^{p} d x \\
& +c\left(f_{B_{R}} 1+|D u|^{p} d x\right)^{2-p}\left(f_{B_{R}} 1+|D \psi|^{p} d x\right)^{p-1} .
\end{aligned}
$$

We omit the proof because it is analogous to the proof of the preceding Lemma 3.6.

\section{Zero order estimates by potentials}

\subsection{Decay estimates for a zero order excess.}

We begin with the following decay estimate at $L^{1}$-level for solutions of homogeneous equations, which follows from classical theory. 
Lemma 4.1. Consider a solution $u \in W^{1, p}\left(B_{R}\right)$ of the elliptic equation

$$
-\operatorname{div} a(x, D u)=0 \quad \text { on } B_{R},
$$

where $0<R \leq 1$, and where the assumptions (1.4) and (1.5) are in force. Then the solution satisfies the decay estimate

$$
\varrho^{1-n} \int_{B_{\varrho}}|D u| d x \leq c\left(\frac{\varrho}{R}\right)^{\beta} f_{B_{R}}\left|u-(u)_{R}\right| d x+c R^{\beta}
$$

for all radii $\varrho \in\left(0, \frac{R}{2}\right]$. Here, the constants $\beta \in(0,1)$ and $c \geq 1$ depend only on $n, p, \nu$ and $L$.

Proof: The standard DeGiorgi-Nash-Moser theory (see [15, Theorem 7.7]) yields the excess decay estimate

$$
\varrho^{p-n} \int_{B_{\varrho}}|D u|^{p} d x \leq c\left(\frac{\varrho}{R}\right)^{p \beta} R^{p-n} \int_{B_{R / 2}}|D u|^{p} d x+c R^{p \beta}
$$

for all radii $\varrho \in\left(0, \frac{R}{2}\right]$, where $\beta \in(0,1)$ and $c \geq 1$ depend only on $n$, $p, \nu$ and $L$. Moreover, testing the equation (4.1) with $\zeta^{p}\left(u-(u)_{R}\right)$, where $\zeta$ denotes a suitable cut-off function, standard estimates yield the well-known Caccioppoli inequality

$$
f_{B_{\sigma}}|D u|^{p} d x \leq c f_{B_{\varrho}} 1+\left|\frac{u-(u)_{R}}{\varrho-\sigma}\right|^{p} d x
$$

for all radii $\sigma, \varrho$ with $\frac{R}{2} \leq \sigma<\varrho \leq R$ (cf. also the proof of Lemma 3.4). We let $p^{\#}:=\frac{n p}{n-p}$ in the case $p<n$ and choose $p^{\#}$ arbitrarily with $p^{\#}>p$ if $p \geq n$. Then, we interpolate the $L^{p}$-norm appearing in the preceding estimate between the $L^{1}$ - and the $L^{p^{\#}}$-norms and apply Young's inequality in order to derive the estimate

$$
\begin{aligned}
f_{B_{\varrho}}\left|\frac{u-(u)_{R}}{\varrho-\sigma}\right|^{p} d x & =\left(\frac{R}{\varrho-\sigma}\right)^{p} f_{B_{\varrho}}\left|\frac{u-(u)_{R}}{R}\right|^{p} d x \\
\leq & \kappa\left(f_{B_{\varrho}}\left|\frac{u-(u)_{R}}{R}\right|^{p^{\#}} d x\right)^{\frac{p}{p^{\#}}} \\
& +c_{\kappa}\left(\frac{R}{\varrho-\sigma}\right)^{\gamma}\left(f_{B_{\varrho}} \frac{\left|u-(u)_{R}\right|}{R} d x\right)^{p}
\end{aligned}
$$

for any $\kappa \in(0,1)$, with an exponent $\gamma>p$, depending only on $n$ and $p$, and a constant $c_{\kappa}$ depending additionally on $\kappa$. By the Minkowski and 
the Poincaré-Sobolev inequality, we have

$$
\begin{aligned}
\left(f_{B_{\varrho}}\left|\frac{u-(u)_{R}}{R}\right|^{p^{\#}} d x\right)^{\frac{1}{p \#}} & \leq\left(f_{B_{\varrho}}\left|\frac{u-(u)_{\varrho}}{R}\right|^{p^{\#}} d x\right)^{\frac{1}{p^{\#}}}+\frac{\left|(u)_{\varrho}-(u)_{R}\right|}{R} \\
& \leq c\left(f_{B_{\varrho}}|D u|^{p} d x\right)^{\frac{1}{p}}+f_{B_{\varrho}} \frac{\left|u-(u)_{R}\right|}{R} d x .
\end{aligned}
$$

Joining the last two estimates and keeping in mind that $\varrho \in\left[\frac{R}{2}, R\right]$, we deduce

$$
f_{B_{\varrho}}\left|\frac{u-(u)_{R}}{\varrho-\sigma}\right|^{p} d x \leq \kappa c f_{B_{\varrho}}|D u|^{p} d x+c_{\kappa}\left(\frac{R}{\varrho-\sigma}\right)^{\gamma}\left(f_{B_{R}} \frac{\left|u-(u)_{R}\right|}{R} d x\right)^{p} .
$$

In view of (4.3), we have thereby established the bound

$$
f_{B_{\sigma}}|D u|^{p} d x \leq \kappa c f_{B_{\varrho}}|D u|^{p} d x+c_{\kappa}\left(\frac{R}{\varrho-\sigma}\right)^{\gamma}\left(f_{B_{R}} \frac{\left|u-(u)_{R}\right|}{R} d x\right)^{p}+c
$$

for all $\frac{R}{2} \leq \sigma<\varrho \leq R$. Choosing $\kappa \in(0,1)$ sufficiently small to guarantee $\kappa c \leq \frac{1}{2}$, we can absorb the first integral on the right-hand side with the help of Lemma 2.2. This leads us to

$$
f_{B_{R / 2}}|D u|^{p} d x \leq c\left(1+f_{B_{R}}\left|\frac{u-(u)_{R}}{R}\right| d x\right)^{p}
$$

Plugging this into (4.2), we arrive at

$$
\varrho^{p-n} \int_{B_{\varrho}}|D u|^{p} d x \leq c\left(\frac{\varrho}{R}\right)^{p \beta}\left(R+f_{B_{R}}\left|u-(u)_{R}\right| d x\right)^{p}+c R^{p \beta}
$$

for all $\varrho \in\left(0, \frac{R}{2}\right]$. From this we derive the claim by bounding the lefthand side from below by Hölder's inequality and keeping in mind that $R \leq 1$.

Before stating the excess decay estimates for the solutions to obstacle problems, we introduce the abbreviation

$$
\Psi\left(B_{R}\left(x_{0}\right)\right):=\int_{B_{R}\left(x_{0}\right)} 1+|D \psi|^{p}+|F|^{p} d x
$$

for all $x_{0} \in \Omega$ and $0<R<\operatorname{dist}\left(x_{0}, \partial \Omega\right)$. With this notation, our result reads as follows. 
Lemma 4.2. Let $u \in L^{1}(\Omega)$ with $u \geq \psi$ a. e. be a limit of approximating solutions for $O P(\psi ; \mu, F)$ with measure data $\mu \in \mathcal{M}_{b}(\Omega)$ and divergence term given by $F \in L^{p}\left(\Omega, \mathbb{R}^{n}\right)$, as in Definition 1.1. Here, we assume that the conditions (1.4) and (1.5) hold for a growth exponent $p>\frac{2 n}{n+1}$. Furthermore, we consider balls $B_{\varrho}\left(x_{0}\right) \subset B_{R / 4}\left(x_{0}\right) \subset B_{R}\left(x_{0}\right) \subset \Omega$. Then we have the following excess decay estimates:

$$
\begin{aligned}
f_{B_{\varrho}\left(x_{0}\right)} \mid u & -(u)_{x_{0}, \varrho} \mid d x \\
\leq & c\left(\frac{\varrho}{R}\right)^{\beta} f_{B_{R}\left(x_{0}\right)}\left|u-(u)_{x_{0}, R}\right| d x+c R^{\beta} \\
& +c\left(\frac{R}{\varrho}\right)^{n}\left\{\left[\frac{|\mu|\left(\overline{B_{R}\left(x_{0}\right)}\right)}{R^{n-p}}\right]^{\frac{1}{p-1}}+\left[\frac{\Psi\left(B_{R}\left(x_{0}\right)\right)}{R^{n-p}}\right]^{\frac{1}{p}}\right\},
\end{aligned}
$$

for $p \geq 2$, while for exponents $\frac{2 n}{n+1}<p<2$, we have

$$
\begin{aligned}
& f_{B_{\varrho}\left(x_{0}\right)} \mid u-(u)_{x_{0}, \varrho} \mid d x+\varrho\left(f_{B_{\varrho}\left(x_{0}\right)}|D u|^{\frac{n}{n+1}} d x\right)^{1+\frac{1}{n}} \\
& \leq c\left(\frac{\varrho}{R}\right)^{\beta} f_{B_{R}\left(x_{0}\right)}\left|u-(u)_{x_{0}, R}\right| d x+c R^{\beta} \\
&+c\left(\frac{R}{\varrho}\right)^{n}\left\{\left[\frac{|\mu|\left(\overline{B_{R}\left(x_{0}\right)}\right)}{R^{n-p}}\right]^{\frac{1}{p-1}}+\left[\frac{\Psi\left(B_{R}\left(x_{0}\right)\right)}{R^{n-p}}\right]^{\frac{1}{p}}\right. \\
&+c R^{2-p}\left(1+f_{B_{R}\left(x_{0}\right)}|D u|^{\frac{n}{n+1}} d x\right)^{\left(1+\frac{1}{n}\right)(2-p)} \\
&\left.\times\left(\frac{|\mu|\left(\overline{B_{R}\left(x_{0}\right)}\right)}{R^{n-p}}+\left[\frac{\Psi\left(B_{R}\left(x_{0}\right)\right)}{R^{n-p}}\right]^{\frac{p-1}{p}}\right)\right\} .
\end{aligned}
$$

Here, $\beta \in(0,1)$ and $c \geq 1$ denote constants that depend at most on $n$, $p, \nu$ and $L$.

Proof: We will prove the claim by a comparison argument consisting of the following steps. 
Step 1: Comparison with the obstacle problem without measure data.

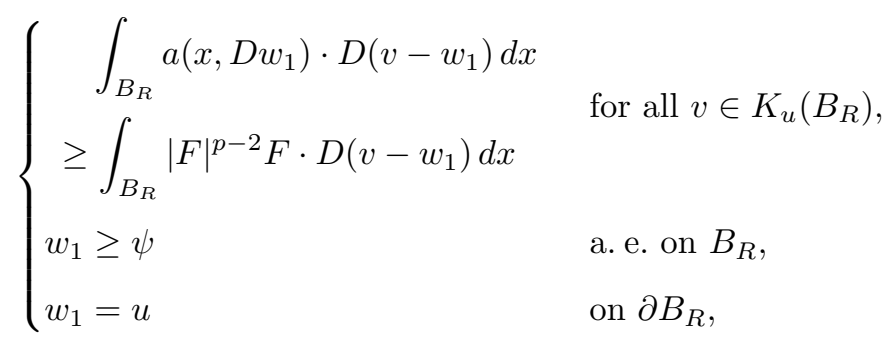

where the space of comparison maps is given by $K_{u}\left(B_{R}\right):=\{v \in u+$ $W_{0}^{1, p}\left(B_{R}\right): v \geq \psi$ a. e. $\}$.

Step 2: Comparison with the obstacle-free problem.

$$
\begin{cases}-\operatorname{div} a\left(x, D w_{2}\right)=-\operatorname{div} a(x, D \psi) & \text { on } B_{R / 2} \\ w_{2}=w_{1} & \text { on } \partial B_{R / 2}\end{cases}
$$

Step 3: Comparison with the homogeneous elliptic equation.

$$
\begin{cases}-\operatorname{div} a\left(x, D w_{3}\right)=0 & \text { on } B_{R / 2} \\ w_{3}=w_{1} & \text { on } \partial B_{R / 2} .\end{cases}
$$

The existence of the solutions to these comparison maps is guaranteed by classical results, see e.g. [27]. Next, we will give the comparison estimates for the three steps listed above.

Step 1: Transition to a problem without measure data. We apply Corollary 3.3 in order to obtain a comparison estimate for $u-w_{1}$. In the case $p \geq 2$, the mentioned corollary in combination with the Poincaré inequality yields

$$
f_{B_{R}}\left|u-w_{1}\right| d x \leq c R f_{B_{R}}\left|D u-D w_{1}\right| d x \leq c\left[\frac{|\mu|\left(\overline{B_{R}}\right)}{R^{n-p}}\right]^{\frac{1}{p-1}}
$$


In the case $\frac{2 n}{n+1}<p<2$, however, the same corollary yields, with the abbreviation $r:=\frac{n}{n+1}$,

$$
\begin{aligned}
& f_{B_{R}}\left|u-w_{1}\right| d x+R\left(f_{B_{R}}\left|D u-D w_{1}\right|^{r} d x\right)^{\frac{1}{r}} \\
& \leq c\left[\frac{|\mu|\left(\overline{B_{R}}\right)}{R^{n-p}}\right]^{\frac{1}{p-1}}+c R^{2-p}\left(f_{B_{R}} 1+|D u|^{r} d x\right)^{\frac{2-p}{r}} \frac{|\mu|\left(\overline{B_{R}}\right)}{R^{n-p}} \\
& \leq c\left[\frac{|\mu|\left(\overline{B_{R}}\right)}{R^{n-p}}\right]^{\frac{1}{p-1}}+c R\left(f_{B_{R}} 1+|D u|^{r} d x\right)^{\frac{1}{r}},
\end{aligned}
$$

with constants $c=c(n, p, \nu)$, where the last estimate follows from Young's inequality with exponents $\frac{1}{2-p}$ and $\frac{1}{p-1}$. As a consequence of the preceding inequality (4.11), we obtain the following estimate for $D w_{1}$, for the case $\frac{2 n}{n+1}<p<2$.

$$
R\left(f_{B_{R}}\left|D w_{1}\right|^{r} d x\right)^{\frac{1}{r}} \leq c\left[\frac{|\mu|\left(\overline{B_{R}}\right)}{R^{n-p}}\right]^{\frac{1}{p-1}}+c R\left(f_{B_{R}} 1+|D u|^{r} d x\right)^{\frac{1}{r}}
$$

For this estimate, we used the elementary inequality $(a+b)^{r} \leq 2^{r}\left(a^{r}+b^{r}\right)$ that holds for all $a, b \geq 0$ and $r>0$. Moreover, according to Lemma 3.4 we have the following reverse Hölder inequality for the solution $w_{1}$ of the problem (4.7).

$$
f_{B_{R / 2}}\left|D w_{1}\right|^{p} d x \leq c\left(f_{B_{R}}\left|D w_{1}\right|^{r} d x\right)^{\frac{p}{r}}+c f_{B_{R}} 1+|D \psi|^{p}+|F|^{p} d x
$$

In view of estimate (4.12), this implies that in the case $\frac{2 n}{n+1}<p<2$ we have

$$
\begin{aligned}
R^{p} f_{B_{R / 2}}\left|D w_{1}\right|^{p} d x \leq & c R^{p}\left(f_{B_{R}} 1+|D u|^{r} d x\right)^{\frac{p}{r}} \\
& +c\left[\frac{|\mu|\left(\overline{B_{R}}\right)}{R^{n-p}}\right]^{\frac{p}{p-1}}+c \frac{\Psi\left(B_{R}\right)}{R^{n-p}} .
\end{aligned}
$$


Step 2: Removing the obstacle. The Comparison Lemma 3.6 gives the estimate

$$
\begin{aligned}
f_{B_{R / 2}}\left|w_{1}-w_{2}\right| d x & \leq c R\left(f_{B_{R / 2}}\left|D w_{1}-D w_{2}\right|^{p} d x\right)^{\frac{1}{p}} \\
& \leq c R\left(f_{B_{R / 2}} 1+|D \psi|^{p}+|F|^{p} d x\right)^{\frac{1}{p}} \\
& \leq c\left[\frac{\Psi\left(B_{R}\right)}{R^{n-p}}\right]^{\frac{1}{p}}
\end{aligned}
$$

in the case $p \geq 2$. For exponents $\frac{2 n}{n+1}<p<2$ however, the same lemma, combined with Poincaré's and Hölder's inequality, yields the estimate

$$
\begin{aligned}
& f_{B_{R / 2}}\left|w_{1}-w_{2}\right| d x+R\left(f_{B_{R / 2}}\left|D w_{1}-D w_{2}\right|^{r} d x\right)^{\frac{1}{r}} \\
& \quad \leq c\left[\frac{\Psi\left(B_{R}\right)}{R^{n-p}}\right]^{\frac{1}{p}}+c\left(R^{p} f_{B_{R / 2}} 1+\left|D w_{1}\right|^{p} d x\right)^{\frac{2-p}{p}}\left[\frac{\Psi\left(B_{R}\right)}{R^{n-p}}\right]^{\frac{p-1}{p}} .
\end{aligned}
$$

Estimating the right-hand side with the help of (4.13), we arrive at

$$
\begin{aligned}
f_{B_{R / 2}}\left|w_{1}-w_{2}\right| d x+R\left(f_{B_{R / 2}}\left|D w_{1}-D w_{2}\right|^{r} d x\right)^{\frac{1}{r}} \\
\leq c\left[\frac{|\mu|\left(\overline{B_{R}}\right)}{R^{n-p}}\right]^{\frac{1}{p-1}}+c\left[\frac{\Psi\left(B_{R}\right)}{R^{n-p}}\right]^{\frac{1}{p}} \\
+c R^{2-p}\left(f_{B_{R}} 1+|D u|^{r} d x\right)^{\frac{2-p}{r}}\left[\frac{\Psi\left(B_{R}\right)}{R^{n-p}}\right]^{\frac{p-1}{p}},
\end{aligned}
$$

where we additionally applied Young's inequality with exponents $\frac{1}{2-p}$ and $\frac{1}{p-1}$. Combining this with the energy estimate (4.12), we deduce

$$
\begin{aligned}
R\left(f_{B_{R / 2}}\left|D w_{2}\right|^{r} d x\right)^{\frac{1}{r}} \leq & c R\left(f_{B_{R}} 1+|D u|^{r} d x\right)^{\frac{1}{r}} \\
& +c\left[\frac{|\mu|\left(\overline{B_{R}}\right)}{R^{n-p}}\right]^{\frac{1}{p-1}}+c\left[\frac{\Psi\left(B_{R}\right)}{R^{n-p}}\right]^{\frac{1}{p}}
\end{aligned}
$$


where we applied Young's inequality once more. Furthermore, we note that according to Lemma 3.5, the map $w_{2}$ satisfies the reverse Hölder inequality

$$
\begin{aligned}
R^{p} f_{B_{R / 2}}\left|D w_{2}\right|^{p} d x \leq & c R^{p}\left(f_{B_{R}}\left|D w_{2}\right|^{r} d x\right)^{\frac{p}{r}}+c R^{p} f_{B_{R}} 1+|D \psi|^{p} d x \\
\leq & c R^{p}\left(f_{B_{R}} 1+|D u|^{r} d x\right)^{\frac{p}{r}} \\
& +c\left[\frac{|\mu|\left(\overline{B_{R}}\right)}{R^{n-p}}\right]^{\frac{p}{p-1}}+c \frac{\Psi\left(B_{R}\right)}{R^{n-p}}
\end{aligned}
$$

where we applied (4.16) for the last estimate.

Step 3: Transition to a homogeneous equation. According to Lemma 3.7 and Poincaré's inequality, we have the following comparison estimate for the case $p \geq 2$.

$$
f_{B_{R / 2}}\left|w_{2}-w_{3}\right| d x \leq c R\left(f_{B_{R / 2}} 1+|D \psi|^{p} d x\right)^{\frac{1}{p}} \leq c\left[\frac{\Psi\left(B_{R}\right)}{R^{n-p}}\right]^{\frac{1}{p}}
$$

In the case $\frac{2 n}{n+2}<p<2$, Lemma 3.7 combined with Poincaré's and Hölder's inequalities yields

$$
\begin{aligned}
f_{B_{R / 2}} \mid & w_{2}-w_{3} \mid d x+R\left(f_{B_{R / 2}}\left|D w_{2}-D w_{3}\right|^{r} d x\right)^{\frac{1}{r}} \\
\leq & c\left[\frac{\Psi\left(B_{R}\right)}{R^{n-p}}\right]^{\frac{1}{p}}+c\left(R^{p} f_{B_{R / 2}} 1+\left|D w_{2}\right|^{p} d x\right)^{\frac{2-p}{p}}\left[\frac{\Psi\left(B_{R}\right)}{R^{n-p}}\right]^{\frac{p-1}{p}} \\
\leq & c\left[\frac{|\mu|\left(\overline{B_{R}}\right)}{R^{n-p}}\right]^{\frac{1}{p-1}}+c\left[\frac{\Psi\left(B_{R}\right)}{R^{n-p}}\right]^{\frac{1}{p}} \\
& +c R^{2-p}\left(f_{B_{R}} 1+|D u|^{r} d x\right)^{\frac{2-p}{r}}\left[\frac{\Psi\left(B_{R}\right)}{R^{n-p}}\right]^{\frac{p-1}{p}} .
\end{aligned}
$$


For the last step, we applied (4.17) and Young's inequality with exponents $\frac{1}{2-p}$ and $\frac{1}{p-1}$.

Final step: Proof of the excess decay estimate. Joining the estimates (4.9), (4.14) and (4.18), we infer in the case $p \geq 2$

$$
f_{B_{R / 2}}\left|u-w_{3}\right| d x \leq c\left[\frac{|\mu|\left(\overline{B_{R}}\right)}{R^{n-p}}\right]^{\frac{1}{p-1}}+c\left[\frac{\Psi\left(B_{R}\right)}{R^{n-p}}\right]^{\frac{1}{p}}
$$

Similarly, in the case $\frac{2 n}{n+1}<p<2$, we combine the estimates (4.10), (4.15) and (4.19) in order to deduce the bound

$$
\begin{aligned}
f_{B_{R / 2}} \mid u & -w_{3} \mid d x+R\left(f_{B_{R / 2}}\left|D u-D w_{3}\right|^{r} d x\right)^{\frac{1}{r}} \\
\leq & c\left[\frac{|\mu|\left(\overline{B_{R}}\right)}{R^{n-p}}\right]^{\frac{1}{p-1}}+c\left[\frac{\Psi\left(B_{R}\right)}{R^{n-p}}\right]^{\frac{1}{p}} \\
& +c R^{2-p}\left(f_{B_{R}} 1+|D u|^{r} d x\right)^{\frac{2-p}{r}}\left(\frac{|\mu|\left(\overline{B_{R}}\right)}{R^{n-p}}+\left[\frac{\Psi\left(B_{R}\right)}{R^{n-p}}\right]^{\frac{p-1}{p}}\right) .
\end{aligned}
$$

Next, we exploit the fact that $w_{3}$ weakly solves the homogeneous equation (4.8), so that by the DeGiorgi-Nash-Moser theory in the form stated in Lemma 4.1, it satisfies the excess decay estimate

$$
\begin{aligned}
& f_{B_{\varrho}}\left|w_{3}-\left(w_{3}\right)_{\varrho}\right| d x+\varrho\left(f_{B_{\varrho}}\left|D w_{3}\right|^{r} d x\right)^{\frac{1}{r}} \\
& \leq c \varrho^{1-n} \int_{B_{\varrho}}\left|D w_{3}\right| d x \\
& \leq c\left(\frac{\varrho}{R}\right)^{\beta} f_{B_{R / 2}}\left|w_{3}-\left(w_{3}\right)_{R / 2}\right| d x+c R^{\beta}
\end{aligned}
$$

for all $\varrho \in\left(0, \frac{R}{4}\right]$, where the exponent $\beta \in(0,1)$ and the constant $c$ both depend only on $n, p, \nu$ and $L$. From this we infer the analogous decay 
estimate for $u$ as follows. For all radii $\varrho \in\left(0, \frac{R}{4}\right]$, we estimate

$$
\begin{aligned}
f_{B_{\varrho}} \mid & u-(u)_{\varrho} \mid d x \\
\leq & f_{B_{\varrho}}\left|w_{3}-\left(w_{3}\right)_{\varrho}\right| d x+2 f_{B_{\varrho}}\left|u-w_{3}\right| d x \\
\leq & c\left(\frac{\varrho}{R}\right)^{\beta} f_{B_{R / 2}}\left|w_{3}-\left(w_{3}\right)_{R / 2}\right| d x+c R^{\beta}+c\left(\frac{R}{\varrho}\right)^{n} f_{B_{R}}\left|u-w_{3}\right| d x \\
& \leq c\left(\frac{\varrho}{R}\right)^{\beta} f_{B_{R}}\left|u-(u)_{R}\right| d x+c R^{\beta}+c\left(\frac{R}{\varrho}\right)^{n} f_{B_{R}}\left|u-w_{3}\right| d x .
\end{aligned}
$$

From this we deduce the claim (4.5) in the case $p \geq 2$ by estimating the last integral by (4.20). In the case $\frac{2 n}{n+1}<p<2$, we can analogously estimate the last integral by (4.21) in order to bound the first term on the left-hand side of the claimed estimate (4.6). However, we additionally need to control the integrals of $|D u|^{r}$. For this we apply (4.22) in order to deduce

$$
\begin{aligned}
\varrho\left(f_{B_{\varrho}}|D u|^{r} d x\right)^{\frac{1}{r}} \leq & c \varrho\left(f_{B_{\varrho}}\left|D w_{3}\right|^{r} d x\right)^{\frac{1}{r}}+c \varrho\left(f_{B_{\varrho}}\left|D u-D w_{3}\right|^{r} d x\right)^{\frac{1}{r}} \\
\leq & c\left(\frac{\varrho}{R}\right)^{\beta} f_{B_{R / 2}}\left|w_{3}-\left(w_{3}\right)_{R / 2}\right| d x+c R^{\beta} \\
& +c\left(\frac{R}{\varrho}\right)^{\frac{n}{r}-1} R\left(f_{B_{R / 2}}\left|D u-D w_{3}\right|^{r} d x\right)^{\frac{1}{r}} \\
\leq & c\left(\frac{\varrho}{R}\right)^{\beta} f_{B_{R}}\left|u-(u)_{R}\right| d x+c R^{\beta} \\
& +c\left(\frac{\varrho}{R}\right)^{\beta} f_{B_{R / 2}}\left|u-w_{3}\right| d x \\
& +c\left(\frac{R}{\varrho}\right)^{n}\left(f_{B_{R / 2}}\left|D u-D w_{3}\right|^{r} d x\right)^{\frac{1}{r}} .
\end{aligned}
$$

Estimating the last two integrals by (4.21), we derive the remaining part of the claim (4.6) and thereby conclude the proof of the lemma. 


\subsection{Pointwise estimates for solutions in terms of potentials.}

We continue to use the notations $\mathbf{W}_{1, p}^{\mu}$ for the Wolff potential and $\mathbf{I}_{p}^{\mu}$ for the Riesz potential as defined in (1.2), respectively in (2.6), and furthermore we use the abbreviation $\Psi$ as introduced in (4.4). With these notations, the zero order potential estimate reads as follows.

Theorem 4.3. There is a constant $c=c(n, p, \nu, L) \geq 1$ such that the following holds. Under the assumptions (1.4) and (1.5), we assume that $u$ is a limit of approximating solutions to $O P(\psi ; \mu, F)$, with measure data $\mu \in \mathcal{M}_{b}(\Omega)$ and divergence term given by $F \in L^{p}\left(\Omega, \mathbb{R}^{n}\right)$, cf. Definition 1.1. Then, in the case $p \geq 2$ the following estimate holds true.

$$
\left|u\left(x_{0}\right)\right| \leq c f_{B_{R}\left(x_{0}\right)} 1+|u| d x+c \mathbf{W}_{1, p}^{\mu}\left(x_{0}, R\right)+c \int_{0}^{R}\left[\frac{\Psi\left(B_{\varrho}\left(x_{0}\right)\right)}{\varrho^{n-p}}\right]^{\frac{1}{p}} \frac{d \varrho}{\varrho}
$$

in every Lebesgue point $x_{0} \in \Omega$ of $u$ and any $0<R<\min \left\{1\right.$, dist $\left.\left(x_{0}, \partial \Omega\right)\right\}$. In the case $\frac{2 n}{n+1}<p<2$, however, we have the bound

$$
\begin{aligned}
\left|u\left(x_{0}\right)\right| \leq & c f_{B_{R}\left(x_{0}\right)} 1+|u| d x+c R\left(f_{B_{R}\left(x_{0}\right)}|D u|^{\frac{n}{n+1}} d x\right)^{1+\frac{1}{n}} \\
& +c \mathbf{W}_{1, p}^{\mu}\left(x_{0}, R\right)+c \int_{0}^{R}\left[\frac{\Psi\left(B_{\varrho}\left(x_{0}\right)\right)}{\varrho^{n-p}}\right]^{\frac{1}{p}} \frac{d \varrho}{\varrho} .
\end{aligned}
$$

Moreover, for $\frac{2 n}{n+1}<p<2$ the following Morrey type estimate holds:

$$
\begin{aligned}
\sup _{0<\varrho \leq R} \varrho\left(f_{B_{\varrho}\left(x_{0}\right)}|D u|^{\frac{n}{n+1}} d x\right)^{1+\frac{1}{n}} \leq & c f_{B_{R}\left(x_{0}\right)} 1+|u| d x \\
& +c R\left(f_{B_{R}\left(x_{0}\right)}|D u|^{\frac{n}{n+1}} d x\right)^{1+\frac{1}{n}} \\
& +c \mathbf{W}_{1, p}^{\mu}\left(x_{0}, R\right) \\
& +c \int_{0}^{R}\left[\frac{\Psi\left(B_{\varrho}\left(x_{0}\right)\right)}{\varrho^{n-p}}\right]^{\frac{1}{p}} \frac{d \varrho}{\varrho} .
\end{aligned}
$$


Remark 4.4. We point out that in the case $p<2$, we can replace the nonlinear Wolff potential $\mathbf{W}_{1, p}^{\mu}$ appearing in the estimate by a power of the linear Riesz potential $\mathbf{I}_{p}^{\mu}$ because of the estimate

$$
\mathbf{W}_{1, p}^{\mu}\left(x_{0}, R\right) \leq\left[\mathbf{I}_{p}^{\mu}\left(x_{0}, 2 R\right)\right]^{\frac{1}{p-1}}
$$

that holds for every $x_{0} \in \Omega$ and $0<R<\frac{1}{2} \operatorname{dist}\left(x_{0}, \partial \Omega\right)$, provided $p<2$. This is a consequence of an elementary inequality for infinite sums, see e. g. [35, Lemma 2.4].

Proof: We fix a Lebesgue point $x_{0} \in \Omega$ of $u$ and choose a parameter $\theta \in$ $\left(0, \frac{1}{4}\right)$ so small that

$$
c \theta^{\beta} \leq \frac{1}{2}
$$

where $c \geq 1$ and $\beta \in(0,1)$ denote the constants determined in Lemma 4.2. In particular, this choice of $\theta$ can be made only in dependence on the data $n, p, \nu$, and $L$. With such a choice of $\theta$, we let $R_{\ell}:=\theta^{\ell} R$ and $B_{\ell}:=B_{R_{\ell}}\left(x_{0}\right)$ for every $\ell \in \mathbb{N}$. For notational convenience, we introduce the abbreviations

$$
\Phi(\varrho):=\left[\frac{|\mu|\left(\overline{B_{\varrho}\left(x_{0}\right)}\right)}{\varrho^{n-p}}\right]^{\frac{1}{p-1}}+\left[\frac{\Psi\left(B_{\varrho}\left(x_{0}\right)\right)}{\varrho^{n-p}}\right]^{\frac{1}{p}}
$$

for every $\varrho \in(0, R]$. We begin with the elementary estimate

$$
\begin{aligned}
\left|(u)_{B_{m+1}}\right| & \leq\left|(u)_{B_{2}}\right|+\sum_{\ell=2}^{m}\left|(u)_{B_{\ell+1}}-(u)_{B_{\ell}}\right| \\
& \leq \frac{1}{\theta^{2 n}} f_{B_{R}\left(x_{0}\right)}|u| d x+\frac{1}{\theta^{n}} \sum_{\ell=2}^{m} f_{B_{\ell}}\left|u-(u)_{B_{\ell}}\right| d x
\end{aligned}
$$

that holds for every $m \in \mathbb{N}$. The remaining part of the proof will be given separately for the cases $p \geq 2$ and $p<2$.

Case 1: $p \geq 2$. In the last sum of (4.26), we can estimate each term by means of the excess decay estimate (4.5) from Lemma 4.2. In view of 
our choice of $\theta$ according to (4.25), this yields

$$
\begin{aligned}
\sum_{\ell=2}^{m} f_{B_{\ell}}\left|u-(u)_{B_{\ell}}\right| d x \leq & \frac{1}{2} \sum_{\ell=1}^{m-1} f_{B_{\ell}}\left|u-(u)_{B_{\ell}}\right| d x \\
& +c \sum_{\ell=1}^{m-1} R_{\ell}^{\beta}+\frac{c}{\theta^{n}} \sum_{\ell=1}^{m-1} \Phi\left(R_{\ell}\right) \\
\leq & \frac{1}{2} \sum_{\ell=2}^{m} f_{B_{\ell}}\left|u-(u)_{B_{\ell}}\right| d x \\
& +c f_{B_{R}\left(x_{0}\right)}|u| d x+c R^{\beta}+c \sum_{\ell=1}^{m-1} \Phi\left(R_{\ell}\right) .
\end{aligned}
$$

Here, we can absorb the first term on the right-hand side, while the last sum can be estimated by the corresponding integral with the help of Lemma 2.3. In this way, we deduce

$$
\sum_{\ell=2}^{m} f_{B_{\ell}}\left|u-(u)_{B_{\ell}}\right| d x \leq c f_{B_{R}\left(x_{0}\right)}|u| d x+c R^{\beta}+c \int_{0}^{R} \Phi(\varrho) \frac{d \varrho}{\varrho}
$$

for all $m \in \mathbb{N}$. Plugging this into (4.26), we arrive at

$$
\left|(u)_{B_{m+1}}\right| \leq c f_{B_{R}\left(x_{0}\right)}|u| d x+c R^{\beta}+c \int_{0}^{R} \Phi(\varrho) \frac{d \varrho}{\varrho}
$$

for all $m \in \mathbb{N}$. Since $x_{0}$ was chosen as a Lebesgue point of $u$, letting $m \rightarrow \infty$ yields the claim.

Case 2: $\frac{2 n}{n+1}<p<2$. The main difference in this case is that we additionally have to control integrals of $|D u|^{r}$, where $r:=\frac{n}{n+1}$, since these terms appear on the right-hand side of the excess decay estimate. Therefore, we iterate the excess decay estimate (4.6) from Lemma 4.2 in the following way, keeping in mind the choice of $\theta$ in (4.25).

$$
\begin{aligned}
\sum_{\ell=2}^{m} f_{B_{\ell}} \mid & -(u)_{B_{\ell}} \mid d x+\sum_{\ell=2}^{m} R_{\ell}\left(f_{B_{\ell}}|D u|^{r} d x\right)^{\frac{1}{r}} \\
\leq & \frac{1}{2} \sum_{\ell=1}^{m-1} f_{B_{\ell}}\left|u-(u)_{B_{\ell}}\right| d x+c \sum_{\ell=1}^{m-1} R_{\ell}^{\beta}+c \sum_{\ell=1}^{m-1} \Phi\left(R_{\ell}\right) \\
& +c \sum_{\ell=1}^{m-1} R_{\ell}^{2-p}\left(1+f_{B_{\ell}}|D u|^{r} d x\right)^{\frac{2-p}{r}} \Phi\left(R_{\ell}\right)^{p-1} .
\end{aligned}
$$


Applying Young's inequality with exponents $\frac{1}{2-p}$ and $\frac{1}{p-1}$ to each term of the last sum, we get

$$
\begin{aligned}
c \sum_{\ell=1}^{m-1} R_{\ell}^{2-p} & \left(1+f_{B_{\ell}}|D u|^{r} d x\right)^{\frac{2-p}{r}} \Phi\left(R_{\ell}\right)^{p-1} \\
\leq & \frac{1}{2} \sum_{\ell=1}^{m-1} R_{\ell}\left(f_{B_{\ell}}|D u|^{r} d x\right)^{\frac{1}{r}}+c \sum_{\ell=1}^{m-1} R_{\ell}+c \sum_{\ell=1}^{m-1} \Phi\left(R_{\ell}\right) .
\end{aligned}
$$

Joining the last two estimates, we arrive at

$$
\begin{aligned}
\sum_{\ell=2}^{m} f_{B_{\ell}} \mid u & -(u)_{B_{\ell}} \mid d x+\sum_{\ell=2}^{m} R_{\ell}\left(f_{B_{\ell}}|D u|^{r} d x\right)^{\frac{1}{r}} \\
\leq & \frac{1}{2} \sum_{\ell=2}^{m} f_{B_{\ell}}\left|u-(u)_{B_{\ell}}\right| d x+\frac{1}{2} \sum_{\ell=2}^{m} R_{\ell}\left(f_{B_{\ell}}|D u|^{r} d x\right)^{\frac{1}{r}} \\
& +c f_{B_{R}\left(x_{0}\right)}|u| d x+c R\left(f_{B_{R}\left(x_{0}\right)}|D u|^{r} d x\right)^{\frac{1}{r}}+c R^{\beta}+c \sum_{\ell=1}^{m-1} \Phi\left(R_{\ell}\right) .
\end{aligned}
$$

Here, the second last line can be absorbed into the left-hand side, and the last sum can be estimated by means of Lemma 2.3. In this fashion, we deduce

$$
\begin{aligned}
\sum_{\ell=2}^{m} f_{B_{\ell}} \mid u & -(u)_{B_{\ell}} \mid d x+\sum_{\ell=2}^{m} R_{\ell}\left(f_{B_{\ell}}|D u|^{r} d x\right)^{\frac{1}{r}} \\
\leq & c f_{B_{R}\left(x_{0}\right)}|u| d x \\
& +c R\left(f_{B_{R}\left(x_{0}\right)}|D u|^{r} d x\right)^{\frac{1}{r}}+c R^{\beta}+c \int_{0}^{R} \Phi(\varrho) \frac{d \varrho}{\varrho} .
\end{aligned}
$$

Combining this with (4.26), we infer

$$
\left|(u)_{B_{m+1}}\right| \leq c f_{B_{R}\left(x_{0}\right)}|u| d x+c R\left(f_{B_{R}\left(x_{0}\right)}|D u|^{r} d x\right)^{\frac{1}{r}}+c R^{\beta}+c \int_{0}^{R} \Phi(\varrho) \frac{d \varrho}{\varrho} .
$$


Keeping in mind that $R \leq 1$ and that $x_{0} \in \Omega$ was chosen as a Lebesgue point of $u$, we infer the claim (4.23) for the case $\frac{2 n}{n+1}<p<2$ by letting $m \rightarrow \infty$. Furthermore, we infer from (4.27)

$$
\begin{aligned}
\sup _{\ell \in \mathbb{N}} R_{\ell}\left(f_{B_{\ell}}|D u|^{r} d x\right)^{\frac{1}{r}} \leq & c f_{B_{R}\left(x_{0}\right)} 1+|u| d x \\
& +c R\left(f_{B_{R}\left(x_{0}\right)}|D u|^{r} d x\right)^{\frac{1}{r}}+c \int_{0}^{R} \Phi(\varrho) \frac{d \varrho}{\varrho},
\end{aligned}
$$

which implies the last claim (4.24). The proof is complete.

Combining the potential estimates from the preceding theorem withthe Lorentz space estimates from Section 2, we infer the following Lorentz space estimates for solutions.

Corollary 4.5. Suppose that $u \in L^{1}(\Omega)$ is a limit of approximating solutions of $\operatorname{OP}(\psi ; \mu, F)$-where the assumptions (1.4) and (1.5) are in force for a growth exponent $p \in\left(\frac{2 n}{n+1}, n\right)$ - and let $1<r<\frac{n}{p}$ and $\max \left\{1, \frac{1}{p-1}\right\} \leq t \leq \infty$.

(i) (Estimates above the duality exponent): In the case $r>\left(p^{*}\right)^{\prime}=$ $\frac{n p}{n p-n+p}$, the assumptions

$$
\mu \in L(r, t) \quad \text { and } \quad|F|^{p-1},|D \psi|^{p-1} \in L\left(\frac{n r}{n-r}, t\right)
$$

imply $|u|^{p-1} \in L\left(\frac{n r}{n-p r}, t\right)\left(\Omega^{\prime}\right)$ for every $\Omega^{\prime} \Subset \Omega$ and the corresponding estimate

$$
\begin{aligned}
\left\||u|^{p-1}\right\|_{L\left(\frac{n r}{n-p r}, t\right)\left(\Omega^{\prime}\right)} \leq & c\left\{1+\|u\|_{L^{1}}+[D u]_{\frac{n}{n+1}}+\|\mu\|_{L(r, t)}\right. \\
& \left.+\left\||F|^{p-1}\right\|_{L\left(\frac{n r}{n-r}, t\right)}+\left\||D \psi|^{p-1}\right\|_{L\left(\frac{n r}{n-r}, t\right)}\right\}
\end{aligned}
$$

with a constant $c=c\left(n, p, \nu, L, \operatorname{dist}\left(\Omega^{\prime}, \partial \Omega\right)\right)$, where the term

$$
[D u]_{\frac{n}{n+1}}:=\left(\int_{\Omega}|D u|^{\frac{n}{n+1}} d x\right)^{1+\frac{1}{n}}
$$

can be omitted in the case $p \geq 2$. 
(ii) (Estimates below the duality exponent): If $r<\left(p^{*}\right)^{\prime}$, under the general assumption $F, D \psi \in L^{p}\left(\Omega, \mathbb{R}^{n}\right)$ the condition $\mu \in L(r, t)$ implies $|u|^{p-1} \in L\left(\frac{n r}{n-p r}, t\right)\left(\Omega^{\prime}\right)$ for every $\Omega^{\prime} \Subset \Omega$ and

$$
\begin{aligned}
\left\||u|^{p-1}\right\|_{L\left(\frac{n r}{n-p r}, t\right)\left(\Omega^{\prime}\right)} \leq c\left(1+\|u\|_{L^{1}}\right. & {[D u]_{\frac{n}{n+1}}+\|\mu\|_{L(r, t)} } \\
& \left.+\|F\|_{L^{p}}^{p-1}+\|D \psi\|_{L^{p}}^{p-1}\right),
\end{aligned}
$$

with $c=c\left(n, p, \nu, L,\left|\Omega^{\prime}\right|, \operatorname{dist}\left(\Omega^{\prime}, \partial \Omega\right)\right)$. Again, the term $[D u]_{\frac{n}{n+1}}$ is superfluous for $p \geq 2$.

Proof: We start with the case $p \geq 2$. From Theorem 4.3 we infer the estimate

$$
\begin{aligned}
\left|u\left(x_{0}\right)\right| \leq & c\left(1+R^{-n}\|u\|_{L^{1}}+\mathbf{W}_{1, p}^{\mu}\left(x_{0}, R\right)\right) \\
& +c \int_{0}^{R}\left[\frac{|F|^{p}\left(B_{\varrho}\left(x_{0}\right)\right)}{\varrho^{n-p}}\right]^{\frac{1}{p}}+\left[\frac{|D \psi|^{p}\left(B_{\varrho}\left(x_{0}\right)\right)}{\varrho^{n-p}}\right]^{\frac{1}{p}} \frac{d \varrho}{\varrho}
\end{aligned}
$$

for every $x_{0} \in \Omega^{\prime}$ and $0<R<\min \left\{1, \operatorname{dist}\left(\Omega^{\prime}, \partial \Omega\right)\right\}$, where $c=c(n, p, \nu, L)$. For the estimate of the Wolff potential of $\mu$, we use the bound (2.7) with $d=1, \gamma=p, q=\frac{1}{p-1} \leq 1$ and $s=t$, which yields

$$
\left\|\mathbf{W}_{1, p}^{\mu}(\cdot, R)\right\|_{L\left(\frac{n r(p-1)}{n-p r}, t(p-1)\right)} \leq\|\mu\|_{L(r, t)}^{\frac{1}{p-1}} .
$$

For the estimate of the integral in (4.30) we proceed differently depending on whether $r$ is larger or smaller than the duality exponent $\left(p^{*}\right)^{\prime}$. In the first case, we have $\frac{n r}{n-r}>p^{\prime}$, which enables us to apply (2.7) with the choices $\tilde{r}=\frac{p-1}{p} \frac{n r}{n-r}>1, s=\frac{t(p-1)}{p}, d=p, \gamma=p$ and $q=\frac{1}{p}$ to the function $F$, with the result

$$
\begin{aligned}
\left\|\int_{0}^{R}\left[\frac{|F|^{p}\left(B_{\varrho}(\cdot)\right)}{\varrho^{n-p}}\right]^{\frac{1}{p}} \frac{d \varrho}{\varrho}\right\|_{L\left(\frac{n r(p-1)}{n-p r}, t(p-1)\right)} & \leq c\|F\|_{L\left(\frac{n r(p-1)}{n-r}, t(p-1)\right)} \\
& =c\left\||F|^{p-1}\right\|_{L\left(\frac{n r}{n-r}, t\right)}^{\frac{1}{p-1}} .
\end{aligned}
$$

At this stage we note that formally, the estimate (2.7) is only applicable if $t \geq p^{\prime}$, but in view of the quasilinearity of the integral on the left-hand side, the Marcinkiewicz interpolation theorem yields the preceding estimate for all $t \geq 1$. The same estimate as above holds for the function $D \psi$ instead of $F$. This concludes the proof of (4.28) in the case $p \geq 2$.

In the case of exponents $r<\left(p^{*}\right)^{\prime}$, however, we apply the borderline estimate (2.8) with the parameters $d=p, \gamma=p$ and $q=\frac{1}{p}$, with the 
result

$$
\left\|\int_{0}^{R}\left[\frac{|F|^{p}\left(B_{\varrho}(\cdot)\right)}{\varrho^{n-p}}\right]^{\frac{1}{p}} \frac{d \varrho}{\varrho}\right\|_{L\left(\frac{n p}{n-p}, \infty\right)} \leq c\|F\|_{L^{p}}
$$

Since the assumption $r<\left(p^{*}\right)^{\prime}$ is equivalent to $\frac{n r(p-1)}{n-p r}<\frac{n p}{n-p}$, we can exploit the continuous embedding

$$
L\left(\frac{n p}{n-p}, \infty\right)\left(\Omega^{\prime}\right) \hookrightarrow L\left(\frac{n r(p-1)}{n-p r}, t(p-1)\right)\left(\Omega^{\prime}\right),
$$

with the result

$$
\left\|\int_{0}^{R}\left[\frac{|F|^{p}\left(B_{\varrho}(\cdot)\right)}{\varrho^{n-p}}\right]^{\frac{1}{p}} \frac{d \varrho}{\varrho}\right\|_{L\left(\frac{n r(p-1)}{n-p r}, t(p-1)\right)\left(\Omega^{\prime}\right)} \leq c\left(\Omega^{\prime}\right)\|F\|_{L^{p}},
$$

with a constant $c\left(\Omega^{\prime}\right)=c\left(n, p, r, \nu, L,\left|\Omega^{\prime}\right|\right)$. The same estimate holds for $D \psi$ instead of $F$, which concludes the proof of (4.29) in the case $p \geq 2$.

The proof in the case $p<2$ is similar. Here, Theorem 4.3 implies by Remark 4.4 that

$$
\begin{aligned}
\left|u\left(x_{0}\right)\right| \leq & c\left(1+R^{-n}\|u\|_{L^{1}}+R^{-n}[D u]_{\frac{n}{n+1}}+\left[\mathbf{I}_{p}^{\mu}\left(x_{0}, 2 R\right)\right]^{\frac{1}{p-1}}\right) \\
& +c \int_{0}^{R}\left[\frac{|F|^{p}\left(B_{\varrho}\left(x_{0}\right)\right)}{\varrho^{n-p}}\right]^{\frac{1}{p}}+\left[\frac{|D \psi|^{p}\left(B_{\varrho}\left(x_{0}\right)\right)}{\varrho^{n-p}}\right]^{\frac{1}{p}} \frac{d \varrho}{\varrho} .
\end{aligned}
$$

The last integral can be estimated in the same way as for $p \geq 2$, while for the Riesz potential, we apply the bound (2.7) in the case $d=1, \gamma=p$ and $q=1$, which yields

$$
\left\|\mathbf{I}_{p}^{\mu}\left(x_{0}, 2 R\right)\right\|_{L\left(\frac{n r}{n-p r}, t\right)} \leq c\|\mu\|_{L(r, t)} .
$$

This completes the proof of the corollary.

\subsection{Continuity of solutions.}

Based on the excess decay estimates from Section 4.1 and the potential estimates from Section 4.2, we are now able to provide conditions on the data that imply boundedness or even continuity of the solutions to obstacle problems with measure data $\mu \in \mathcal{M}_{b}(\Omega)$. This is the subject of the following theorem.

Theorem 4.6. We assume that for a growth exponent $p>\frac{2 n}{n+1}$, the conditions (1.4) and (1.5) are satisfied for a structure function $a: \Omega \times$ $\mathbb{R}^{n} \rightarrow \mathbb{R}^{n}$. We consider a limit of approximating solutions $u \in L^{1}(\Omega)$ with $u \geq \psi$ of the obstacle problem $O P(\psi ; \mu, F)$ with measure data $\mu \in$ $\mathcal{M}_{b}(\Omega)$, divergence term given by $F \in L^{p}\left(\Omega, \mathbb{R}^{n}\right)$, and an obstacle function $\psi \in W^{1, p}(\Omega)$. 
(i) If for every subdomain $\Omega^{\prime} \Subset \Omega$, the data satisfy

$$
\sup _{x_{0} \in \Omega^{\prime}}\left\{\mathbf{W}_{1, p}^{\mu}\left(x_{0}, R\right)+\int_{0}^{R}\left[\varrho^{p-n} \int_{B_{\varrho}\left(x_{0}\right)}|D \psi|^{p}+|F|^{p} d x\right]^{\frac{1}{p}} \frac{d \varrho}{\varrho}\right\}<\infty
$$

for some $0<R<\operatorname{dist}\left(\Omega^{\prime}, \partial \Omega\right)$ then we have $u \in L_{\mathrm{loc}}^{\infty}(\Omega)$.

(ii) Assume that additionally to the assumption in (i), we have

$$
\lim _{\varrho \searrow 0} \sup _{x_{0} \in \Omega^{\prime}} \varrho^{p-n}\left[|\mu|\left(B_{\varrho}\left(x_{0}\right)\right)+\int_{B_{\varrho}\left(x_{0}\right)}|D \psi|^{p}+|F|^{p} d x\right]=0,
$$

for every subdomain $\Omega^{\prime} \Subset \Omega$. Then we have $u \in \operatorname{VMO}_{\text {loc }}(\Omega) \cap$ $L_{\mathrm{loc}}^{\infty}(\Omega)$.

(iii) If for every subdomain $\Omega^{\prime} \Subset \Omega$, the property

(4.33) $\lim _{R \searrow 0} \sup _{x_{0} \in \Omega^{\prime}}\left\{\mathbf{W}_{1, p}^{\mu}\left(x_{0}, R\right)+\int_{0}^{R}\left[\varrho^{p-n} \int_{B_{\varrho}\left(x_{0}\right)}|D \psi|^{p}+|F|^{p} d x\right]^{\frac{1}{p}} \frac{d \varrho}{\varrho}\right\}=0$ is satisfied, then $u \in C_{\mathrm{loc}}^{0}(\Omega)$.

In view of the implication (2.9), we infer in particular a sufficient condition for continuity in terms of Lorentz spaces.

Corollary 4.7. For a growth exponent $p \in\left(\frac{2 n}{n+1}, n\right)$, we assume that (1.4) and (1.5) are in force and that $u \in L^{1}(\Omega)$ is a limit of approximating solutions to $\mathrm{OP}(\psi ; \mu, F)$ with measure data $\mu \in \mathcal{M}_{b}(\Omega)$, divergence term given by $F \in L^{p}\left(\Omega, \mathbb{R}^{n}\right)$, and an obstacle function $\psi \in W^{1, p}(\Omega)$. Then the conditions

$$
\mu \in L\left(\frac{n}{p}, \frac{1}{p-1}\right) \quad \text { and } \quad|D \psi|,|F| \in L(n, 1)
$$

imply $u \in C_{\mathrm{loc}}^{0}(\Omega)$.

Proof of the theorem: We fix an arbitrary subdomain $\Omega^{\prime} \Subset \Omega$ and choose another subdomain $\Omega^{\prime \prime}$ with $\Omega^{\prime} \Subset \Omega^{\prime \prime} \Subset \Omega$. The proof is divided into three steps, corresponding to the three statements of the theorem.

Step 1: Local boundedness. We use the assumption (4.31) on the subdomain $\Omega^{\prime \prime}$ in order to infer from Theorem 4.3 that

$$
M_{0}:=\sup _{y \in \Omega^{\prime \prime}}|u(y)|<\infty .
$$

Since $\Omega^{\prime} \subset \Omega$ was chosen arbitrarily and $\Omega^{\prime \prime} \supset \Omega^{\prime}$, this implies the claim $u \in L_{\mathrm{loc}}^{\infty}(\Omega)$ and thus concludes the proof of part (i) of the theorem. 
Moreover, we infer from estimate (4.24) in Theorem 4.3 that

$$
M_{1}:=\sup _{\varrho>0} \sup _{y \in \Omega^{\prime}} \varrho\left(f_{B_{\varrho}(y) \cap \Omega}|D u|^{r} d x\right)^{\frac{1}{r}}<\infty
$$

holds in the case $\frac{2 n}{n+1}<p<2$, where we abbreviated $r:=\frac{n}{n+1}$.

Step 2: VMO-regularity. We claim that

$$
\mathbf{V}(R):=\sup _{0<\varrho \leq R} \sup _{y \in \Omega^{\prime}} f_{B_{\varrho}(y)}\left|u-(u)_{y, \varrho}\right| d x \longrightarrow 0 \quad \text { as } R \searrow 0
$$

which implies the claim $u \in \operatorname{VMO}_{\text {loc }}(\Omega)$ in view of the arbitrariness of $\Omega^{\prime} \Subset \Omega$. For the proof of the claim (4.36), we fix an arbitrary $\varepsilon>0$. For a radius $0<R_{0}<\min \left\{1, \operatorname{dist}\left(\Omega^{\prime}, \Omega \backslash \Omega^{\prime \prime}\right)\right\}$ to be chosen small later, we let

$$
\delta\left(R_{0}\right):=\sup _{0<\varrho \leq R_{0}} \sup _{y \in \Omega^{\prime}} \varrho^{p-n}\left[|\mu|\left(B_{\varrho}(y)\right)+\int_{B_{\varrho}(y)}|D \psi|^{p}+|F|^{p} d x\right],
$$

noting that by assumption (4.32), the value of $\delta \geq 0$ can be made arbitrarily small by choosing $R_{0}>0$ appropriately, and we assume without loss of generality that $\delta \leq 1$.

Case 1: $p \geq 2$. Because of (4.34) and (4.37), the excess decay estimate from Lemma 4.2 implies

$$
f_{B_{\varrho}(y)}\left|u-(u)_{y, \varrho}\right| d x \leq c\left(\frac{\varrho}{R}\right)^{\beta} M_{0}+c R_{0}^{\beta}+c\left(\frac{R}{\varrho}\right)^{n} \delta\left(R_{0}\right)^{\frac{1}{p}}
$$

for all $y \in \Omega^{\prime}, R \leq R_{0}$ and $\varrho \in\left(0, \frac{R}{4}\right)$. Here, the constant $\beta \in(0,1)$ appearing in the above estimate is determined by Lemma 4.2 in dependence on $n, p, \nu$ and $L$, and the constant $c$ depends on the same data. We want to apply the preceding bound for a radius $\varrho:=\theta R$, where $\theta \in\left(0, \frac{1}{4}\right)$. By choosing first $\theta \in\left(0, \frac{1}{4}\right)$ and then $0<R_{0}<\min \left\{1, \operatorname{dist}\left(\Omega^{\prime}, \Omega \backslash \Omega^{\prime \prime}\right)\right\}$ sufficiently small, we can ensure

$$
c \theta^{\beta} M_{0} \leq \frac{\varepsilon}{2} \quad \text { and } \quad c R_{0}^{\beta}+c \theta^{-n} \delta\left(R_{0}\right)^{\frac{1}{p}} \leq \frac{\varepsilon}{2} .
$$


With these choices of parameters, we infer from (4.38) that

$$
f_{B_{\theta R}(y)}\left|u-(u)_{y, \theta R}\right| d x \leq \varepsilon
$$

holds for all $R \in\left(0, R_{0}\right)$ and all $y \in \Omega^{\prime}$. By the definition of $\mathbf{V}$ in (4.36), this implies $\mathbf{V}\left(\theta R_{0}\right) \leq \varepsilon$. Since $\varepsilon>0$ was chosen arbitrarily, this concludes the proof of (4.36) and thereby of the claim (ii) in the case $p \geq 2$.

Case 2: $\frac{2 n}{n+1}<p<2$. In this case, we apply (4.35) additionally to (4.34) and (4.37), in order to infer from the excess decay estimate in Lemma 4.2 that

$$
\begin{aligned}
f_{B_{\varrho}(y)} & \left|u-(u)_{y, \varrho}\right| d x+\varrho\left(f_{B_{\varrho}(y)}|D u|^{r} d x\right)^{\frac{1}{r}} \\
& \leq c\left(\frac{\varrho}{R}\right)^{\beta} M_{0}+c R_{0}^{\beta}+c\left(\frac{R}{\varrho}\right)^{n} \delta\left(R_{0}\right)^{\frac{1}{p}}+c\left(1+M_{1}\right)^{2-p} \delta\left(R_{0}\right)^{\frac{p-1}{p}}
\end{aligned}
$$

holds for all $y \in \Omega^{\prime}, R \leq R_{0}$ and $\varrho \in\left(0, \frac{R}{4}\right)$. Similarly as above, we can now choose first $\theta \in\left(0, \frac{1}{4}\right)$ and then $0<R_{0}<\min \left\{1, \operatorname{dist}\left(\Omega^{\prime}, \Omega \backslash \Omega^{\prime \prime}\right)\right\}$ small enough to guarantee

$$
f_{B_{\theta R}(y)}\left|u-(u)_{y, \theta R}\right| d x+\theta R\left(f_{B_{\theta R}(y)}|D u|^{r} d x\right)^{\frac{1}{r}} \leq \varepsilon
$$

for all $y \in \Omega^{\prime}$ and $R \in\left(0, R_{0}\right)$. This implies in particular $\mathbf{V}(\theta R) \leq \varepsilon$ and therefore the claimed VMO-regularity $u \in \mathrm{VMO}_{\text {loc }}(\Omega)$. Moreover, we deduce

$$
\sup _{0<\varrho \leq R} \sup _{y \in \Omega^{\prime}} \varrho\left(f_{B_{\varrho}(y)}|D u|^{r} d x\right)^{\frac{1}{r}} \longrightarrow 0 \quad \text { as } R \searrow 0
$$

in the case $\frac{2 n}{n+1}<p<2$.

Step 3: Continuity. For all $y \in \Omega^{\prime}$ and $0<\varrho<\operatorname{dist}(y, \partial \Omega)$, we write

$$
\Phi(y, \varrho):=\left[\frac{|\mu|\left(\overline{B_{\varrho}(y)}\right)}{\varrho^{n-p}}\right]^{\frac{1}{p-1}}+\left[\varrho^{p-n} \int_{B_{\varrho}(y)}|D \psi|^{p}+|F|^{p} d x\right]^{\frac{1}{p}} .
$$

We choose a parameter $\theta \in\left(0, \frac{1}{4}\right)$ small enough in order to achieve

$$
c \theta^{\beta} \leq \frac{1}{2}
$$

where $c \geq 1$ and $\beta \in(0,1)$ denote the constants determined in Lemma 4.2, and abbreviate $R_{\ell}:=\theta^{\ell} R$ and $B_{\ell}(y):=B_{R_{\ell}}(y)$ for all $\ell \in \mathbb{N}_{0}$. Our aim 
is to prove that $(u)_{B_{\ell}(y)}$ is a uniform Cauchy sequence, which implies that its limit $u(y)$ depends continuously on $y$, as desired. To this end, we estimate for any $k, m \in \mathbb{N}$ with $k<m$

$$
\left|(u)_{B_{m}}-(u)_{B_{k}}\right| \leq \sum_{\ell=k}^{m-1}\left|(u)_{B_{\ell+1}}-(u)_{B_{\ell}}\right| \leq \frac{1}{\theta^{n}} \sum_{\ell=k}^{m-1} f_{B_{\ell}}\left|u-(u)_{B_{\ell}}\right| d x
$$

For the remainder of the proof, we distinguish once more between the cases $p \geq 2$ and $p<2$.

Case 1: $p \geq 2$. We apply the excess decay estimate from Lemma 4.2 to each term in the last sum of (4.41), which yields in view of our choice of $\theta$ in (4.40)

$$
\begin{aligned}
\sum_{\ell=k}^{m-1} f_{B_{\ell}}\left|u-(u)_{B_{\ell}}\right| d x \leq & \frac{1}{2} \sum_{\ell=k}^{m-2} f_{B_{\ell}}\left|u-(u)_{B_{\ell}}\right| d x \\
& +\frac{1}{2} f_{B_{k-1}}\left|u-(u)_{B_{k-1}}\right| d x \\
& +c \sum_{\ell=k-1}^{m-2} R_{\ell}^{\beta}+\frac{c}{\theta^{n}} \sum_{\ell=k-1}^{m-2} \Phi\left(y, R_{\ell}\right) .
\end{aligned}
$$

After absorbing the first term from the right-hand side, estimating the last series by means of Lemma 2.3 and plugging the resulting estimate into (4.41), we deduce

$$
\left|(u)_{B_{m}(y)}-(u)_{B_{k}(y)}\right| \leq f_{B_{k-1}(y)}\left|u-(u)_{B_{k-1}(y)}\right| d x+c R_{k-1}^{\beta}+c \int_{0}^{R_{k-2}} \Phi(y, \varrho) \frac{d \varrho}{\varrho}
$$

for all $y \in \Omega^{\prime}$ and $k<m$ in $\mathbb{N}$. Here, the first term on the right-hand side vanishes in the limit $k, m \rightarrow \infty$ independently from $y \in \Omega^{\prime}$, because of the local VMO-regularity established in step 2, while the last term converges to zero uniformly in $y \in \Omega^{\prime}$, according to assumption (4.33). We deduce that $(u)_{B_{k}(y)}$ is a uniform Cauchy sequence and thus converges to a continuous limit map. But by Lebesgue's differentiation theorem, this limit map equals $u(y)$ a. e., which implies the claimed continuity of $u$ in the case $p \geq 2$. 
Case 2: $\frac{2 n}{n+1}<p<2$. In this case, the application of Lemma 4.2 yields the estimate

$$
\begin{aligned}
\sum_{\ell=k}^{m-1} f_{B_{\ell}} \mid u & -(u)_{B_{\ell}} \mid d x+\sum_{\ell=k}^{m-1} R_{\ell}\left(f_{B_{\ell}}|D u|^{r} d x\right)^{\frac{1}{r}} \\
\leq & \frac{1}{2} \sum_{\ell=k}^{m-2} f_{B_{\ell}}\left|u-(u)_{B_{\ell}}\right| d x \\
& +\frac{1}{2} f_{B_{k-1}}\left|u-(u)_{B_{k-1}}\right| d x+c \sum_{\ell=k-1}^{m-2} R_{\ell}^{\beta}+\frac{c}{\theta^{n}} \sum_{\ell=k-1}^{m-2} \Phi\left(y, R_{\ell}\right) \\
& +\frac{c}{\theta^{n}} \sum_{\ell=k-1}^{m-2} R_{\ell}^{2-p}\left(1+f_{B_{\ell}}|D u|^{r} d x\right)^{\frac{2-p}{r}} \Phi\left(y, R_{\ell}\right)^{p-1},
\end{aligned}
$$

where $r:=\frac{n}{n+1}$. Applying Young's inequality with exponents $\frac{1}{2-p}$ and $\frac{1}{p-1}$ to the terms of the last sum, we infer

$$
\begin{aligned}
\sum_{\ell=k}^{m-1} f_{B_{\ell}} \mid u & -(u)_{B_{\ell}} \mid d x+\sum_{\ell=k}^{m-1} R_{\ell}\left(f_{B_{\ell}}|D u|^{r} d x\right)^{\frac{1}{r}} \\
\leq & \frac{1}{2} \sum_{\ell=k}^{m-1} f_{B_{\ell}}\left|u-(u)_{B_{\ell}}\right| d x \\
& +\frac{1}{2} \sum_{\ell=k}^{m-1} R_{\ell}\left(f_{B_{\ell}}|D u|^{r} d x\right)^{\frac{1}{r}} \\
& +\frac{1}{2} f_{B_{k-1}}\left|u-(u)_{B_{k-1}}\right| d x \\
& +\frac{1}{2} R_{k-1}\left(f_{B_{k-1}}|D u|^{r} d x\right)^{\frac{1}{r}}+c R_{k-1}^{\beta}+c \sum_{\ell=k-1}^{m-2} \Phi\left(y, R_{\ell}\right) .
\end{aligned}
$$

Here, we can re-absorb the second line into the left-hand side. Note that this is possible since $|D u|^{r} \in L^{1}(\Omega)$ holds for the range of exponents $p>\frac{2 n}{n+1}$, see Remark 1.2. Estimating furthermore the last sum by means of Lemma 2.3 and plugging the resulting estimate into (4.41), we 
arrive at

$$
\begin{aligned}
\left|(u)_{B_{m}(y)}-(u)_{B_{k}(y)}\right| \leq & f_{B_{k-1}(y)}\left|u-(u)_{B_{k-1}(y)}\right| d x \\
& +R_{k-1}\left(f_{B_{k-1}(y)}|D u|^{r} d x\right)^{\frac{1}{r}} \\
& +c R_{k-1}^{\beta}+c \int_{0}^{R_{k-2}} \Phi(y, \varrho) \frac{d \varrho}{\varrho}
\end{aligned}
$$

for all $y \in \Omega^{\prime}$ and $k<m$ in $\mathbb{N}$. Here, the first two integrals on the righthand side uniformly tend to zero as $k \rightarrow \infty$ by the VMO-regularity established in step 2, respectively by (4.39), and the last term in the above estimate uniformly tends to zero because of our assumption (4.33). This and Lebesgue's differentiation theorem imply the uniform convergence $(u)_{B_{k}(y)} \rightarrow u(y)$ as $k \rightarrow \infty$, which yields the claimed continuity of $u$.

\subsection{Hölder continuity.}

Lemma 4.8. We consider a limit of approximating solutions $u \in L^{1}(\Omega)$ to the obstacle problem $\mathrm{OP}(\psi ; \mu, F)$, where the assumptions (1.4) and (1.5) are in force for a growth exponent $p>\frac{2 n}{n+1}$ and the data satisfy the Morrey estimate

$$
\begin{aligned}
& \sup _{x_{0} \in \Omega^{\prime}} \sup _{0<\varrho<1}\left[\varrho^{p-n-\gamma(p-1)}|\mu|\left(B_{\varrho}\left(x_{0}\right) \cap \Omega\right)\right. \\
&\left.+\varrho^{p-n-\gamma p} \int_{B_{\varrho}\left(x_{0}\right) \cap \Omega}|F|^{p}+|D \psi|^{p} d x\right]<\infty
\end{aligned}
$$

for some $\gamma \in(0,1)$ and every subdomain $\Omega^{\prime} \Subset \Omega$. Let $\beta=\beta(n, p, \nu, L) \in$ $(0,1)$ denote the exponent from Lemma 4.1. Then we have $u \in C_{\mathrm{loc}}^{0, \alpha}(\Omega)$ for every $0<\alpha<\min \{\beta, \gamma, \gamma(p-1)\}$.

Proof: We fix an arbitrary domain $\Omega^{\prime} \Subset \Omega$ and choose a domain $\Omega^{\prime \prime}$ with $\Omega^{\prime} \Subset \Omega^{\prime \prime} \Subset \Omega$. For $\varrho_{0}:=\min \left\{1, \operatorname{dist}\left(\Omega^{\prime \prime}, \partial \Omega\right)\right\}$, we abbreviate

$$
K:=\sup _{x_{0} \in \Omega^{\prime \prime}} \sup _{0<\varrho<\varrho_{0}} \varrho^{-\gamma}\left\{\left[\frac{|\mu|\left(B_{\varrho}\left(x_{0}\right)\right)}{\varrho^{n-p}}\right]^{\frac{1}{p-1}}+\left[\varrho^{p-n} \int_{B_{\varrho}\left(x_{0}\right)}|F|^{p}+|D \psi|^{p} d x\right]^{\frac{1}{p}}\right\}
$$


which is finite by assumption. The zero order potential estimates from Theorem 4.3 therefore imply the following bound in the case $\frac{2 n}{n+1}<p<2$

$$
\begin{aligned}
M_{1} & :=\sup _{x_{0} \in \Omega^{\prime}} \sup _{0<\varrho<\varrho_{0}} \varrho\left(f_{B_{\varrho}\left(x_{0}\right)}|D u|^{\frac{n}{n+1}} d x\right)^{1+\frac{1}{n}} \\
& \leq c\left(\|u\|_{L^{1}}+\left(\int_{\Omega}|D u|^{\frac{n}{n+1}} d x\right)^{1+\frac{1}{n}}+K+1\right)<\infty .
\end{aligned}
$$

We point out that the integral of $|D u|^{\frac{n}{n+1}}$ is finite for $p>\frac{2 n}{n+1}$, cf. Remark 1.2. We define the excess functional

$$
E\left(x_{0}, \varrho\right):=f_{B_{\varrho}\left(x_{0}\right)}\left|u-(u)_{x_{0}, \varrho}\right| d x
$$

for every $x_{0} \in \Omega^{\prime}$ and $0<\varrho<\varrho_{0}$. Moreover, we fix a Hölder exponent $0<\alpha<\min \{\beta, \gamma, \gamma(p-1)\}$ and choose a parameter $\theta \in(0,1)$ with $c \theta^{\beta} \leq \theta^{\alpha}$, where $c$ denotes the constant from Lemma 4.2. Applying this lemma with radii $R<\varrho_{0}$ and $\varrho=\theta R$ and keeping in mind the definitions of $M_{1}$ and $K$, we deduce

$$
E\left(x_{0}, \theta R\right) \leq \theta^{\alpha} E\left(x_{0}, R\right)+c R^{\beta}+c R^{\gamma} K
$$

in the case $p \geq 2$, while for $\frac{2 n}{n+1}<p<2$, the same lemma gives

$$
E\left(x_{0}, \theta R\right) \leq \theta^{\alpha} E\left(x_{0}, R\right)+c R^{\beta}+c R^{\gamma} K+c\left(1+M_{1}\right)^{2-p}\left(R^{\gamma} K\right)^{p-1},
$$

where here, $c=c(\alpha, n, p, \nu, L)$. In both cases, we thus get an estimate of the type

$$
E\left(x_{0}, \theta R\right) \leq \theta^{\alpha} E\left(x_{0}, R\right)+\tilde{c} R^{\min \{\beta, \gamma, \gamma(p-1)\}}
$$

with $\tilde{c}=\tilde{c}\left(n, p, \nu, L, \alpha, \gamma, \Omega^{\prime}, M_{1}, K\right)$. Since $\alpha<\min \{\beta, \gamma, \gamma(p-1)\}$, the repeated application of this estimate leads us to

$$
E\left(x_{0}, \theta^{k} R\right) \leq \theta^{\alpha k}\left[E\left(x_{0}, R\right)+\tilde{c}\right]
$$

for all $k \in \mathbb{N}$ and $x_{0} \in \Omega^{\prime}$, and finally

$$
f_{B_{r}\left(x_{0}\right)}\left|u-(u)_{x_{0}, r}\right| d x \leq \tilde{c}\left(\frac{r}{R}\right)^{\alpha}\left[\|u\|_{L^{1}}+1\right] .
$$

In view of Campanato's integral characterization of Hölder continuous functions, we established the desired Hölder continuity $u \in C^{0, \alpha}\left(\Omega^{\prime}\right)$, which completes the proof. 


\section{References}

[1] D. R. Adams and N. G. Meyers, Thinness and Wiener criteria for non-linear potentials, Indiana Univ. Math. J. 22 (1972/73), 169-197.

[2] P. Bénilan, L. Boccardo, T. Gallouët, R. Gariepy, M. Pierre, and J. L. VÁzquez, An $L^{1}$-theory of existence and uniqueness of solutions of nonlinear elliptic equations, Ann. Scuola Norm. Sup. Pisa Cl. Sci. (4) 22(2) (1995), 241-273.

[3] A. Bensoussan and J.-L. Lions, "Applications of variational inequalities in stochastic control", Translated from the French, Studies in Mathematics and its Applications 12, North-Holland Publishing Co., Amsterdam-New York, 1982.

[4] L. Boccardo and G. R. Cirmi, Existence and uniqueness of solution of unilateral problems with $L^{1}$ data, J. Convex Anal. 6(1) (1999), 195-206.

[5] L. Boccardo and T. Gallouët, Nonlinear elliptic and parabolic equations involving measure data, J. Funct. Anal. 87(1) (1989), 149-169. DOI : 10.1016/0022-1236(89) 90005-0.

[6] L. Boccardo and T. Gallouët, Problèmes unilatéraux avec données dans $L^{1}$, C. R. Acad. Sci. Paris Sér. I Math. 311(10) (1990), 617-619.

[7] L. Boccardo and T. Gallouët, Nonlinear elliptic equations with right-hand side measures, Comm. Partial Differential Equations 17(3-4) (1992), 641-655. DOI: 10.1080/03605309208820857.

[8] L. Boccardo, T. Galloü̈t, and L. Orsina, Existence and uniqueness of entropy solutions for nonlinear elliptic equations with measure data, Ann. Inst. H. Poincaré Anal. Non Linéaire 13(5) (1996), 539-551.

[9] L. Boccardo and F. Murat, Almost everywhere convergence of the gradients of solutions to elliptic and parabolic equations, Nonlinear Anal. 19(6) (1992), 581-597. DOI: 10.1016/0362546X (92) 90023-8.

[10] G. Dal Maso, F. Murat, L. Orsina, and A. Prignet, Renormalized solutions of elliptic equations with general measure data, Ann. Scuola Norm. Sup. Pisa Cl. Sci. (4) 28(4) (1999), 741-808.

[11] F. Duzaar and G. Mingione, Gradient estimates via nonlinear potentials, Amer. J. Math. 133(4) (2011), 1093-1149. DOI: 10.1353/ajm. 2011.0023. 
[12] F. Duzaar And G. Mingione, Gradient continuity estimates, Calc. Var. Partial Differential Equations 39(3-4) (2010), 379-418. DOI : $10.1007 / \mathrm{s} 00526-010-0314-6$.

[13] F. DuzaAr And G. Mingione, Local Lipschitz regularity for degenerate elliptic systems, Ann. Inst. H. Poincaré Anal. Non Linéaire 27(6) (2010), 1361-1396. DOI: 10.1016/j.anihpc.2010. 07.002.

[14] F. Duzana and G. Mingione, Gradient estimates via linear and nonlinear potentials, J. Funct. Anal. 259(11) (2010), 2961-2998. DOI : $10.1016 / \mathrm{j} \cdot \mathrm{jfa} .2010 .08 .006$.

[15] E. Giusti, "Direct methods in the calculus of variations", World Scientific Publishing Co., Inc., River Edge, NJ, 2003. DOI: 10.1142/ 9789812795557.

[16] J. Heinonen, T. Kilpeläinen, and O. Martio, "Nonlinear potential theory of degenerate elliptic equations", Oxford Mathematical Monographs, Oxford Science Publications, The Clarendon Press, Oxford University Press, New York, 1993.

[17] T. Kilpeläinen And P. Lindqvist, On the Dirichlet boundary value problem for a degenerate parabolic equation, SIAM J. Math. Anal. 27(3) (1996), 661-683. DOI: 10.1137/0527036.

[18] T. KilpeläInen and J. MalÝ, Degenerate elliptic equations with measure data and nonlinear potentials, Ann. Scuola Norm. Sup. Pisa Cl. Sci. (4) 19(4) (1992), 591-613.

[19] T. Kilpeläınen And J. MalÝ, The Wiener test and potential estimates for quasilinear elliptic equations, Acta Math. 172(1) (1994), 137-161. DOI: $10.1007 / \mathrm{BF} 02392793$.

[20] T. KilpeläInen And X. Zhong, Growth of entire $\mathcal{A}$-subharmonic functions, Ann. Acad. Sci. Fenn. Math. 28(1) (2003), 181-192.

[21] D. Kinderlehrer And G. Stampacchia, "An introduction to variational inequalities and their applications", Pure and Applied Mathematics 88, Academic Press, Inc. [Harcourt Brace Jovanovich, Publishers], New York-London, 1980.

[22] J. Kinnunen And P. Lindqvist, Pointwise behaviour of semicontinuous supersolutions to a quasilinear parabolic equation, Ann. Mat. Pura Appl. (4) 185(3) (2006), 411-435. DOI : 10.1007/s10231005-0160-x.

[23] T. KuUsi And G. Mingione, A surprising linear type estimate for nonlinear elliptic equations, C. R. Math. Acad. Sci. Paris 349(15-16) (2011), 889-892. DOI: 10.1016/j.crma.2011.07.025. 
[24] T. Kuusi and G. Mingione, Linear potentials in nonlinear potential theory, Preprint.

[25] C. Leone, Existence and uniqueness of solutions for nonlinear obstacle problems with measure data, Nonlinear Anal., Ser. A: Theory Methods 43(2) (2001), 199-215. DOI: 10.1016/S0362-546X(99) 00190-X.

[26] P. Lindqvist, On the definition and properties of $p$-superharmonic functions, J. Reine Angew. Math. 365 (1986), 67-79. DOI : 10.1515/ crll.1986.365.67.

[27] J.-L. Lions, "Quelques méthodes de résolution des problèmes aux limites non linéaires", Dunod; Gauthier-Villars, Paris, 1969.

[28] J. MalÝ AND W. P. Ziemer, "Fine regularity of solutions of elliptic partial differential equations", Mathematical Surveys and Monographs 51, American Mathematical Society, Providence, RI, 1997.

[29] V. G. MAZ'JA AND V. P. HAVIN, A nonlinear potential theory, Uspehi Mat. Nauk 27(6) (1972), 67-138; English translation in: Russian Math. Surveys 27(6) (1972), 71-148 (1974).

[30] G. Mingione, Gradient potential estimates, J. Eur. Math. Soc. (JEMS) 13(2) (2011), 459-486. DOI: 10.4171/JEMS/258.

[31] P. Oppezzi And A. M. Rossi, Unilateral problems with measure data, Nonlinear Anal., Ser. A: Theory Methods 43(8) (2001), 1057-1088. DOI : 10.1016/S0362-546X (99) 00244-8.

[32] C. Scheven, Existence and gradient estimates in nonlinear problems with irregular obstacles, Habilitation thesis (2011).

[33] C. Scheven, Existence of localizable solutions to nonlinear parabolic problems with irregular obstacles, Preprint (2011).

[34] C. Scheven, Potential estimates for solutions to parabolic obstacle problems, Ann. Acad. Sci. Fenn. Math. (to appear). DOI: 10.5186/aasfm. 2012.3730.

[35] C. Scheven, Gradient potential estimates in nonlinear elliptic obstacle problems with measure data, J. Funct. Anal. 262(6) (2012), 2777--2832. DOI: 10.1016/j.jfa.2012.01.003.

[36] N. S. Trudinger And X.-J. Wang, On the weak continuity of elliptic operators and applications to potential theory, Amer. J. Math. 124(2) (2002), 369-410. DOI: 10.1353/ajm.2002.0012.

[37] W. P. ZIEMER, "Weakly differentiable functions", Sobolev spaces and functions of bounded variation, Graduate Texts in Mathematics 120, Springer-Verlag, New York, 1989. 
Department Mathematik

Universität Erlangen-Nürnberg

Cauerstraße 11

91058 Erlangen

Germany

E-mail address: scheven@mi.uni-erlangen.de

Primera versió rebuda el 8 d'agost de 2011, darrera versió rebuda el 13 de febrer de 2012. 